\title{
COMPUTING A CHIEF SERIES AND THE SOLUBLE RADICAL OF A MATRIX GROUP OVER A FINITE FIELD
}

\author{
DEREK F. HOLT AND MARK J. STATHER
}

\begin{abstract}
We describe an algorithm for computing a chief series, the soluble radical, and two other characteristic subgroups of a matrix group over a finite field, which is intended for matrix groups that are too large for the use of base and strong generating set methods. The algorithm has been implemented in MAGMA by the second author.
\end{abstract}

\section{Introduction}

One of the major projects in computational group theory during the past 15 years has been the development of effective algorithms for analysing the structure of linear groups defined by generating matrices over a finite field. The methods that are currently used by default in the GAP and MAGMA systems are based on an extension of the base and strong generating set (BSGS) techniques that have proved so effective for computing in finite permutation groups. These are unfortunately impractical for large groups of matrices, particularly those that involve the classical groups in their natural representations. (See Chapter 4 and Section 7.8.1 of [17] for definitions and details of the BSGS approach to computing in permutation and matrix groups.)

Leedham-Green and O'Brien [23] have implemented procedures in MAGMA that use methods based on a theorem of Aschbacher, which we shall state in Subsection 1.2, to construct a composition tree in a matrix group over a finite field, which effectively identifies the composition factors of the group. See also [29] for a recent survey of this project.

For further structural computations in matrix groups, such as finding Sylow subgroups, a chief series is more useful. As has been demonstrated for various types of computations in finite permutation groups (the calculation of maximal subgroups [9], of automorphism groups [8], and of conjugacy classes of elements [10], for example), a chief series that passes through the characteristic subgroups $O_{\infty}(G)$, $\operatorname{soc}^{*}(G)$, and $\operatorname{Pker}(G)$ of $G$ is particularly expedient. These subgroups are defined as follows.

(i) $O_{\infty}(G)$ is the soluble radical - that is, the largest normal soluble subgroup of $G$.

(ii) $\operatorname{soc}^{*}(G)$ is the complete inverse image in $G$ of the nonabelian socle of $G / O_{\infty}(G)$. So $\operatorname{soc}^{*}(G) / O_{\infty}(G)$ is a direct product of nonabelian simple groups, which are permuted under the conjugation action of $G / O_{\infty}(G)$. 
(iii) $\operatorname{Pker}(G)$ is the complete inverse image in $G$ of the kernel of the permutation action of $G / O_{\infty}(G)$ on the simple factors of $\operatorname{soc}^{*}(G) / O_{\infty}(G)$ described in (ii). So $\operatorname{soc}^{*}(G) \leqslant \operatorname{Pker}(G)$.

Note that $\operatorname{Pker}(G) / \operatorname{soc}^{*}(G)$ is isomorphic to a subgroup of the direct product of the outer automorphism groups of the simple direct factors of $\operatorname{soc}^{*}(G) / O_{\infty}(G)$ which, by the Schreier conjecture, is soluble. Note also that the top factor $G / \operatorname{Pker}(G)$ is isomorphic to a permutation group on the factors of $\operatorname{soc}^{*}(G) / O_{\infty}(G)$, which in practice will be of moderately low degree.

It is the object of this paper to describe algorithms to construct such a series. They have been implemented in MAgma by the second author, and a more detailed description of these methods and of applications to further structural computations in matrix groups can be found in his $\mathrm{PhD}$ thesis [31]. See also [32] for an application to the computation of Sylow subgroups. For an input group $G \leqslant \operatorname{GL}(n, q)$, it is also possible to use the data structures associated with the chief series computed for $G$ to test whether an arbitrary element $g$ of $\operatorname{GL}(n, q)$ lies in $G$, and if so to write $g$ as a word in the generators of $G$.

The computations are carried out in three stages. The first step is to compute a series of normal subgroups of the given group $G$ in which the quotients are either soluble groups for which a polycyclic presentation (see, for example, [17, Chapter 8]) is known, or direct products of isomorphic nonabelian simple groups. The second step, which is comparatively straightforward, is to refine the series to a chief series of the group. The final step is to compute a new chief series passing through $O_{\infty}(G)$, $\operatorname{soc}^{*}(G)$ and $\operatorname{Pker}(G)$. This is achieved by a sequence of operations, each of which interchanges two adjacent chief factors in the series.

We believe our computation of a chief series of $G$ to be about equally efficient on average, in terms of use of both time and space resources, as the computation of a composition tree using the algorithm of Leedham-Green and O'Brien. As we shall see later, there are some places in our algorithm where we are forced to carry out computations in larger groups than should be necessary, and here we will lose ground in the comparison with the composition tree. In other places, however, we save time by avoiding the unnecessary repetition of certain computations in subgroups that are conjugate to other subgroups in which these computations have already been carried out. We shall indicate instances of these two phenomena in the examples described in Section 4.

In the remainder of this introductory section we shall describe some relevant basic algorithms, and state the Aschbacher Theorem. In Section 2 we describe the computation of a chief series of a matrix group $G$, in Section 3 we explain how to rearrange the chief series to make it pass through the three characteristic subgroups of $G$ defined above, and then in Section 4 we present some timings of the second author's MAGMA implementation.

\subsection{Some underlying algorithms}

An algorithm to compute the order of an invertible $d \times d$ matrix is described in [12]. Many of the algorithms of the Matrix Group Recognition Project, and indeed of computational group theory in general, require the construction of random elements of a group. An algorithm to produce uniformly distributed random elements of a finite group is given by Babai [5]. Unfortunately this is too slow 
to be of practical use. For the purposes of an implementation we use the Product Replacement Algorithm [11]. See also [17, Section 3.2.2] for a brief description.

We shall assume throughout that we are able to perform standard computations, including finding chief series, etc., in permutation groups and in soluble groups defined by a polycyclic presentation (see, for example [17, Chapter 8]). The most sophisticated of the algorithms that we shall assume to be available are the Constructive Recognition algorithms for the finite nonabelian simple groups.

We formulate the definition of a constructive recognition algorithm following that of Seress [30, Chapter 8]. However we shall adapt the definition slightly to give emphasis on matrix groups. Recall that $G$ is said to be quasisimple if $G$ is perfect and $G / Z(G)$ is simple, where $Z(G)$ is the centre of $G$. We are interested in the case where a quasisimple group $G$ acts absolutely irreducibly, and so $Z(G)$ is the group of scalar matrices $Z(\operatorname{GL}(n, q)) \cap G$ of $G$.

Definition 1.1. Let $G=\langle X\rangle$ be a group such that either $G$ is a simple permutation group, or else $G$ is a quasisimple absolutely irreducible matrix group over a finite field. Then we define a constructive recognition algorithm for $G$ to be one that is able to do the following:

(i) Find the standard name of the simple group $G / Z(G)$.

(ii) Find a new generating set $Y$ of size $O(\log |G|)$ for $G$, along with words over $X$ for each $y \in Y$, and a presentation of length $O\left(\log ^{2}|G|\right)$ of $G / Z(G)$ on $Y$. (By a word over $X$, we mean a word in $\left(X \cup X^{-1}\right)^{*}$.)

(iii) Compute an epimorphism $\phi$ from $G$ to $S$ where $S$ is the standard copy of $G / Z(G)$, with the property that images and inverse images of elements under $\phi$ can be computed efficiently. (We explain the term standard copy below.)

(iv) Given $g$ in the full symmetric or general linear group of which $G$ is a subgroup, determine whether $g \in G$ and, if so, write $g$ as a word over $Y$.

A non-constructive recognition algorithm is one that can solve part (i) only. A polynomial time Monte-Carlo algorithm for part (i) has been implemented in Magma by Malle and O'Brien. This works roughly by calculating orders of random elements of the group and finding the finite simple group having the same distribution of element orders. See [29, Section 6] for a fuller account.

Observe that, although we assumed at the outset that $G$ is quasisimple, after carrying out this process, we have proved that $G / Z(G)$ is isomorphic to the simple group named in (i) above, and hence we have verified that our assumption was correct. (If we need to verify that $G$ is perfect, then we can do so by choosing the generators in $Y$ to be words lying in $[G, G]$.) This is important because, in our applications to be described later, we are sometimes highly confident but not absolutely certain that $G / Z(G)$ is simple and so we need to verify this assumption.

In (iii), the standard copy of a nonabelian simple group $G$ is a specific group $H$ that depends only on the isomorphism type of $G$, where $H$ is either a primitive permutation group with $H \cong G$, or a quasisimple absolutely irreducible matrix group with $H / Z(H) \cong G$. For example, for $G \cong \operatorname{Alt}(n)$ we choose $H=\operatorname{Alt}(n)$, and for $G \cong \operatorname{PSL}(n, q)$ we choose $H=\mathrm{SL}(n, q)$. We assume also (as in the condition (iv) for $G$ ) that we can test arbitrary elements of the $\operatorname{group} \operatorname{Sym}(n)$ or $\operatorname{GL}(n, q)$ that contains $H$ for membership of $H$. 
The algorithm referred to in part (iv) is known as a solution to the rewriting problem in $G$ on $Y$. In practice we do not store the words that arise as ordinary words over $Y$, which would be impractically long in many cases, but use straight line programs (see, for example [17, Section 3.1.3]) as a more compact storage method. Note that, since from (ii) we can express the elements of $Y$ as words over the original generators $X$, we can also express arbitrary group elements as words (or rather straight line programs) over $X$.

Constructive recognition algorithms for the simple groups is currently an active area of research. The alternating groups can be dealt with by the algorithm of Bratus and Pak [6], which has been implemented in MAGma by Holt. Methods for the classical groups are described in [21]. However, these algorithms have a factor of $q$ in their complexity, making them impractical over large fields. Alternative algorithms are currently under development by Leedham-Green and O'Brien. They rely on the special cases $\operatorname{SL}(2, q)$ and $\operatorname{SL}(3, q)$ which have been dealt with in $[\mathbf{1 3}]$ and [27] respectively. Algorithms for the Suzuki and Ree groups have been developed by Bäärnhielm in [3] and [2] respectively. Many sporadic groups can be dealt with using the Ryba algorithm described in [16].

\subsection{Aschbacher's Theorem}

The major result upon which the Matrix Group Recognition Project is based is a theorem by Aschbacher [1] on the subgroup structure of the general linear group, which we shall now paraphrase.

THEOREM $1.2([\mathbf{1}])$. Let $V$ be the vector space of row vectors on which $\operatorname{GL}(n, q)$ acts, let $G$ be a subgroup of $\mathrm{GL}(n, q)$, and let $Z$ be the group of scalar matrices of $G$. Then one of the following is true.

\section{C1. G acts reducibly.}

C2. $G$ acts imprimitively: $G$ preserves a decomposition of $V$ as a direct sum $V_{1} \oplus$ $V_{2} \oplus \cdots \oplus V_{r}$ of $r>1$ subspaces of dimension $s$, which are permuted transitively by $G$, and so $G \leqslant \mathrm{GL}(s, q) i \operatorname{Sym}(r)$.

C3. $G$ acts on $V$ as a group of semilinear automorphisms of a space of dimension $n /$ e over the extension field $\mathbb{F}_{q^{e}}$ for some $e>1$, and so $G$ embeds in $\Gamma \mathrm{L}\left(\frac{n}{e}, q^{e}\right)$. (This covers the class of 'absolutely reducible' matrix groups where $G$ embeds in $\operatorname{GL}\left(\frac{n}{e}, q^{e}\right)$.)

C4. $G$ preserves a decomposition of $V$ as a tensor product $U \otimes W$ of spaces of dimensions $n_{1}, n_{2}>1$ over $\mathbb{F}_{q}$. Then $G$ is a subgroup of the central product of $\mathrm{GL}\left(n_{1}, q\right)$ and $\mathrm{GL}\left(n_{2}, q\right)$.

C5. $G$ is definable modulo scalars over a subfield: for some proper subfield $\mathbb{F}_{q^{\prime}}$ of $\mathbb{F}_{q}, G^{g} \leqslant \mathrm{GL}\left(n, q^{\prime}\right) . Z$ for some $g \in G L(n, q)$.

C6. For some prime $r, n=r^{m}$ and $G$ is contained in the normaliser of an extraspecial group of order $r^{2 m+1}$ or of a group of order $2^{2 m+2}$ and of symplectic type.

C7. $G$ is tensor-induced : it preserves a decomposition of $V$ as $V_{1} \otimes V_{2} \otimes \cdots \otimes V_{m}$, where each $V_{i}$ has dimension $r>1$ and the set of $V_{i}$ is permuted transitively by $G$, and so $G / Z \leqslant \operatorname{PGL}(r, q)$ i $\operatorname{Sym}(m)$.

C8. $G$ contains and normalises a classical group in its natural representation.

C9. $G$ is almost simple modulo scalars: for some nonabelian simple group $T$ we have $T \leqslant G / Z \leqslant \operatorname{Aut}(T)$. 
In short this theorem states that either $G$ is almost simple modulo scalars (in $\mathrm{C} 8$ or $\mathrm{C} 9$ ) or else preserves some natural geometric structure. If $G$ lies in $\mathrm{C} 1-\mathrm{C} 7$ then we obtain a natural geometric directly computable homomorphism from $G$ to some "smaller" group. We define a directly computable homomorphism to be one that can be evaluated without solving the rewriting problem in $G$; the action of a matrix on an invariant subspace, for example.

Many papers have now been published that describe algorithms and their implementations that recognise groups that lie in one or other of the Aschbacher classes. We shall cite these when we come to describe our procedures for handling groups that lie in these classes in Subsection 2.2.

\section{Computing a chief series}

\subsection{Sequences of homomorphisms}

As we explained in the introduction, our first aim is to construct a normal series in our input group $G \leqslant \operatorname{GL}(n, q)$ in which the quotients are either soluble groups or direct products of isomorphic nonabelian simple groups. This series will be represented by a sequence of homomorphisms.

Definition 2.1. We define a preliminary sequence for $G \leqslant \operatorname{GL}(n, q)$ to be a sequence $\left(\phi_{1}, \ldots, \phi_{k}\right)$ of homomorphisms and a descending sequence $G=K_{0} \geqslant K_{1} \geqslant$ $K_{2} \cdots \geqslant K_{k} \geqslant 1$ of normal subgroups of $G$ that satisfy the following properties:

(i) $K_{i-1} \unlhd \operatorname{Domain}\left(\phi_{i}\right) \leqslant \mathrm{GL}(n, q)$ for $1 \leqslant i \leqslant k$.

(ii) $\operatorname{ker}\left(\phi_{i}\right) \cap K_{i-1}=K_{i}$ for $1 \leqslant i \leqslant k$.

(iii) For each $i, \operatorname{im}\left(\phi_{i}\right)$ is either a soluble group defined by a polycyclic presentation or a direct product of isomorphic nonabelian simple groups.

(iv) $\operatorname{im}\left(\phi_{k}\right)$ is abelian and $Z_{G} \leqslant K_{k-1}$, where $Z_{G}$ is the scalar subgroup of $G$.

(v) $K_{k}=O_{p}(G)$, where $p$ is the defining characteristic of $G$.

In addition, we shall say that the sequence is correct on scalars if $Z_{G} O_{p}(G)=$ $K_{k-1}$ in (iv).

We denote the sequence $\left(\phi_{1}, \ldots, \phi_{k}\right)$ by $[\phi]^{(k)}$. Condition (ii) simply defines the subgroups $K_{i}$ from the maps $\phi_{i}$, so we can refer to $[\phi]^{(k)}$ as a preliminary sequence for $G$ if the remaining conditions are satisfied. In fact we will almost always have $G=\operatorname{Domain}\left(\phi_{1}\right)$ - the only exception is when we adjoin some scalars to $G$ in AlmostSimplemaps below - but the remaining $\phi_{i}$ will frequently be defined on a larger domain than $K_{i-1}$. (This is a potential source of inefficiency in our overall procedure, because we are only interested in the restriction of $\phi_{i}$ to $K_{i-1}$, but we may on occasion waste effort in analysing the action of $\phi_{i}$ on a larger domain.)

Note that Condition (v) says that we insist that $O_{p}(G)$ occurs at the bottom of the normal series for $G$. This subgroup can only be nontrivial for reducible groups. Condition (iv) says that the scalar subgroup $Z_{G}$ of $G$ is also pushed to the bottom of the series, but above $O_{p}(G)$. We allow the final map $\phi_{k}$ to be trivial if $Z_{G}=1$.

The first step in the algorithm is to construct the maps $[\phi]^{(k)}$. This is done by means of a recursive algorithm based on Aschbacher decompositions. The maps $\phi_{i}$ in the sequence will all be directly computable homomorphisms, as defined in Subsection 1.2. They will either be maps associated with Aschbacher decompositions, or will involve other straightforward computations, such as determinants. 
The calculation and identification of the kernels and images, which will be discussed in Subsection 2.3, is only carried out after all of the maps $\phi_{i}$ have been defined. Note that some of the layers $K_{i-1} / K_{i}$ in the series could turn out to be trivial.

The properties $(\mathrm{i})-(\mathrm{v})$ listed will be satisfied by $[\phi]^{(k)}$ provided that all of our procedures work correctly. As we shall see, some of them have a small probability of returning incorrect answers, but these will be detected either when we identify the images and kernels, or when we verify the correctness of the complete series. It is the restriction of $\phi_{i}$ to $K_{i-1}$ that will be used to define and work with the quotient $K_{i-1} / K_{i}$ of the series. Condition (i) ensures that the domain of $\phi_{i}$ is large enough to ensure that we can evaluate it on $K_{i-1}$.

Another point to note is that we often represent insoluble images of the $\phi_{i}$ as direct products of quasisimple groups and work modulo scalars. For example, an image that is really isomorphic to $\operatorname{PSL}(n, q)^{d}$ for some $n, q, d$ would typically be represented as $\operatorname{SL}(n, q)^{d}$ in its natural representation as a subgroup of $\operatorname{SL}(d n, q)$. In such cases, $\phi_{i}$ is represented in the implementation by a map $\tilde{\phi}_{i}$ with codomain $\mathrm{SL}(d n, q)$, for which $\phi_{i}(g)=\tilde{\phi}_{i}(g) Z(\operatorname{SL}(d n, q))$ for $g \in \operatorname{Domain}\left(\phi_{i}\right)$.

In general, a map $\psi: G \rightarrow \operatorname{GL}(n, q)$ for which the induced composite map $\bar{\psi}: G \rightarrow \operatorname{PGL}(n, q)$ is a homomorphism is known as a projective homomorphism or a homomorphism mod scalars. We define the kernel $\operatorname{ker}(\psi)$ of a projective homomorphism to be the kernel of the induced homomorphism to $\operatorname{PGL}(n, q)$, and the image $\operatorname{im}(\psi)$ to be the complete inverse image in $\operatorname{GL}(n, q)$ of the image in $\operatorname{PGL}(n, q)$ of $\bar{\psi}$.

We fix some general notation regarding arbitrary sequences of homomorphisms. Given two sequences $[\phi]^{(k)}$ and $[\psi]^{(m)}$, we shall denote the concatenation of the two sequences by $[\phi]^{(k)}$ cat $[\psi]^{(m)}$. We may also append a single homomorphism $\zeta$ to a sequence and denote the result by $[\phi]^{(k)}$ cat $\zeta$. We denote the pruned sequence $\left(\phi_{1}, \ldots, \phi_{k-1}\right)$ by $[\phi]^{(k-1)}$

Let $\psi: G \rightarrow H$ be an epimorphism for a matrix group $H$, and let $[\phi]^{(k)}$ be a preliminary sequence for $H$. Then we define the pullback PULlBACK $\left([\phi]^{(k)}, \psi\right)$ of $[\phi]^{(k)}$ through $\psi$ to be the sequence $[\zeta]^{(k)}$ with $\zeta_{i}=\phi_{i} \circ \psi$ for each $i$. Then $\operatorname{PullBACK}\left([\phi]^{(k-1)}, \psi\right)$ represents a normal series for the quotient $G / \operatorname{ker}(\psi) \cong H$ of $G$.

We shall also use the pullback construction for projective homomorphisms $\psi$ : $G \rightarrow \mathrm{GL}(d, r)$. In that case, let $H=\operatorname{im}(\psi)$ and let $[\phi]^{(k)}$ be a preliminary sequence for $H$. Then, for $1 \leqslant i \leqslant k-1$, we have $Z_{H} \leqslant \operatorname{ker}\left(\phi_{i}\right)$, and so the composites $\overline{\phi_{i}} \circ \psi(1 \leqslant i \leqslant k-1)$ are (genuine) homomorphisms. We shall denote this sequence of composites by PullbaCK $\left([\phi]^{(k-1)}, \psi\right)$. If, in addition, $H$ acts irreducibly (so $O_{p}(H)=1$ ) and the preliminary sequence $[\phi]^{(k)}$ for $H$ is correct on scalars, then $\operatorname{PullbaCK}\left([\phi]^{(k-1)}, \psi\right)$ will represent a normal series for the quotient $G / \operatorname{ker}(\psi)$ of $G$.

If $\phi_{i}: G \rightarrow H_{i}$ are homomorphisms for $1 \leqslant i \leqslant k$, then we denote by $\phi_{1} \times \cdots \times \phi_{k}$ the homomorphism from $G$ to $H_{1} \times \cdots \times H_{k}$ with $g \mapsto\left(\phi_{1}(g), \ldots, \phi_{k}(g)\right)$.

Our algorithm to compute a preliminary sequence $[\phi]^{(k)}$ for $G$ is described in Subsection 2.2. In Subsection 2.3, we explain how the images and kernels of these maps are computed using a Monte Carlo algorithm that may underestimate their orders. This series is verified and (if required) corrected by constructing a presentation of $G$, turning the whole process into a Las Vegas algorithm. The verification 
process is described in Subsection 2.4, where we also explain how to test membership of arbitrary elements of $\operatorname{GL}(n, q)$ in the subgroups $K_{i}$ in the series. So, in particular, this gives us a membership test for $K_{0}=G$.

The remainder of the normal series from $O_{p}(G)$ to 1 is computed by exploiting the module structure of $G$ on its lower triangular blocks (Subsection 2.5). The normal series for $G$ is then refined into a chief series (Subsection 2.6).

\subsection{Constructing the preliminary sequence of maps}

The main procedure to be described in this subsection is NormaLSERIESMAPS, which constructs a preliminary sequence for a matrix group $G$. This is the part of the procedure for finding a chief series that differs most from the composition tree method of Leedham-Green and O'Brien, so we shall treat this topic in more detail than the other parts of the procedure, and provide pseudocode for the analysis of the groups in each of the nine Achbacher classes.

We shall assume that a version of this algorithm is available for permutation groups. This is dealt with by the BSGS techniques described by Cannon and Holt in [7] and implemented in MAGMA. Since a permutation group has no defining characteristic or scalar subgroup, we define the final map $\phi_{k}$ in a preliminary sequence for a permutation group to have trivial domain and image, and we do not insist that $\operatorname{im}\left(\phi_{k-1}\right)$ is abelian.

We shall not write out the pseudocode for NormalSERIESMAPS itself, since it simply uses the methods discussed in Section 1 to find an Aschbacher decomposition, and then calls the appropriate subroutine for that type of decomposition. As we explained in Section 1, there are programs available that test $G$ for membership in each of the Aschbacher classes $\mathrm{C} 1-\mathrm{C} 8$. When they find such a decomposition they return any associated directly computable homomorphisms, such as the action of a matrix on a proper subspace in the reducible case. If the tests fail to find a decomposition in any of these classes, then we assume that the group is in Class C9, in which case it is almost simple modulo scalars and is not equal to a classical group in its natural representation.

Unfortunately, several of these tests, including the tests for classes C2, C4, C5, $\mathrm{C} 6$ and $\mathrm{C} 7$, may occasionally fail to reach a decision within a reasonable time. In that case, the group is almost always in Class C9 even if it is in one of the other classes as well, so our policy is to abort the test and, provided that none of the other tests returns a positive answer, to assume that the group is in C9. This means that there is a small danger that this assumption is false, and then the group might even be falsely identified as being a specific almost simple group. The error would however be discovered when we come to perform constructive recognition of the (nonabelian) simple composition factors, which will be discussed in Subsection 2.4.

We remark also that, as we shall see later, the correctness of the complete procedure requires us to test for classes $\mathrm{C} 1, \mathrm{C} 2$ and $\mathrm{C} 3$ before $\mathrm{C} 4, \mathrm{C} 5$ and $\mathrm{C} 7$, and to test for $\mathrm{C} 4$ before C6. Errors in which, for example, we test for and find that $G$ lies in $\mathrm{C} 4$ after having failed to detect that it also lies in $\mathrm{C} 2$ are extremely rare (in fact we have never known them to occur), but they would be detected when we applied NormalSERIESMAPS recursively to the actions on the tensor factors.

We shall now describe each of the procedures for the individual Aschbacher classes that are called by NORMALSERIESMAPS. These procedures generally call NORMALSERIESMAPS recursively on a permutation group, or on a matrix group of 
smaller dimension or over a smaller field than $G$. In fact all of these recursive calls are to permutation groups or to irreducible matrix groups, and so the final map $\phi_{k}$ in the preliminary sequence returned by any of these calls will have trivial kernel. Indeed, the only procedure that can return a final map $\phi_{k}$ with nontrivial kernel $O_{p}(G)$ is Reduciblemaps.

We claim that, provided that no errors are made in the execution of these procedures (as explained above, certain types of errors may occur with small probability, and these would be detected later), the sequence of homomorphisms returned by each of the procedures is a preliminary sequence for $G$. Furthermore we claim that the procedures that process Aschbacher decompositions in the classes C4-C9 will return preliminary sequences that are correct on scalars. These properties need to be proved as part of the correctness proofs of the procedures, and it may be assumed by induction that they are true for all recursive calls made within the procedures. Having said that, we shall not in fact write out formal correctness proofs, but we shall of course point out any aspects of them that may not be clear.

Throughout, we refer to the natural $\mathbb{F}_{q} G$-module of row vectors by $M_{G}$, and the vector space of row vectors by $V_{G}$. Given any $G$-module (or vector space) $M$ we define ActionGroup $(M)$ to be the group generated by the action of the generators of $G$ on $M$ and $\operatorname{ACTION}(M)$ to be the homomorphism that maps $g \in G$ to its action on $M$. We also use ProjectiveAction $(M)$ to denote an induced projective homomorphism; this arises for groups that preserve tensor product and induced tensor product decompositions.

We shall denote the scalar subgroup of a matrix group $G$ by $Z_{G}$ and we define the function $\operatorname{ScAlarMaP}(n, q)$ to be the isomorphism from $Z_{\mathrm{GL}(n, q)} \rightarrow \mathbb{F}_{q}^{\times}$with $\mathbb{F}_{q}^{\times}$represented by a polycyclic presentation. In the procedures to calculate a preliminary sequence for groups in the Aschbacher classes $\mathrm{C} 4-\mathrm{C} 9$, we shall define the final map in the sequence to be $\operatorname{ScAlarMap}(n, q)$. As we shall see, this will ensure that the returned preliminary sequence is correct on scalars. This is necessary for the correctness of the complete procedure. The preliminary sequences defined for groups in classes $\mathrm{C} 1, \mathrm{C} 2$, and $\mathrm{C} 3$ will not necessarily be correct on scalars.

All of the procedures take as input a subgroup $G$ of $\operatorname{GL}(n, q)$ and output a preliminary sequence for $G$. The input group is assumed to lie in the Aschbacher class for that procedure, and in some cases it is assumed not to lie in various other Aschbacher classes. As we have already mentioned, for all procedures other than ReducibleMaps, we assume that $G$ is not in Class C1. Other assumptions of this type will be justified as they arise.

\section{Reduciblemaps $(G)$}

Assume: $G$ lies in Class C1.

1 Construct (using the Meataxe) a composition series $0=E_{m}<\cdots<E_{1}<E_{0}=M_{G}$ for $M_{G}$

2 for $i \in[1 \ldots m]$

3 do $A_{i}:=\operatorname{ActionGroup}\left(E_{i-1} / E_{i}\right) ; \psi_{i}:=\operatorname{Action}\left(E_{i-1} / E_{i}\right)$;

6 return $\left[\zeta^{(1)}\right]^{\left(k_{1}-1\right)}$ cat $\cdots$ cat $\left[\zeta^{(m)}\right]^{\left(k_{m}-1\right)}$ cat $\left(\zeta_{k_{1}}^{(1)} \times \cdots \times \zeta_{k_{m}}^{(m)}\right)$; 
Notice that we push the scalars coming from each of the irreducible actions of $G$ on $E_{i-1} / E_{i}$ to the bottom of the preliminary sequence returned for $G$. So the image of the final map in the sequence returned will be abelian, and its domain will contain $Z_{G}$, possibly as a proper subgroup.

Our policy of analysing, via the recursive call to NORMALSERIESMAPS, each of the action groups of $E_{i-1} / E_{i}$ separately appears to be necessary in order to obtain a normal series for $G$, but in some examples it can result in our repeating the analyses of the same chief factors of $G$. This will happen, roughly speaking, when the action of $G$ on the $E_{i-1} / E_{i}$ is diagonal rather than a full direct product. We could, in principle, avoid such repetition if some of the $E_{i-1} / E_{i}$ were isomorphic as $G$-modules, but we have not yet attempted this in the implementation.

We turn now to groups in Class C2. Notice at Line 7 in the procedure below, we are applying NoRMALSERIESMAPS recursively only to the action of the stabilizer of the first block in the decomposition on that block. The corresponding actions on the other blocks are computed by conjugation in Line 12. This is a potentially significant gain in efficiency compared with the composition tree program of Leedham-Green and O'Brien [23], in which the kernels of the actions on all of the blocks are analysed separately.

The maps $\zeta_{i}$ in Line 12 will not necessarily be surjective even when $\psi_{i}$ is surjective, but the images will be subdirect products of the images of the $\psi_{i}$ and so, in the insoluble case, will still be direct products of isomorphic simple groups. (See Proposition 2.9 and the associated discussion in Subsection 2.3 below.)

\section{$\operatorname{IMPRIMITIVEMAPS}(G)$}

Assume: $G$ lies in Class C2 but not in Class C1.

1 Construct (using methods described in [19]) a set of blocks of imprimitivity $\Omega=\left\{V_{1}, \ldots, V_{r}\right\}$ for $G$, along with a homomorphism $\rho: G \rightarrow \operatorname{Sym}(\Omega) ;$

$2 \quad \Sigma:=\operatorname{im}(\rho)$;

$3[\tau]^{(k)}:=\operatorname{Pullback}(\operatorname{NormalSeriesmaps}(\Sigma), \rho)$;

$4 G_{1}:=G_{V_{1}}$ the stabiliser of $V_{1}$ in $G$;

5 Let $M_{1}$ be $V_{1}$ considered as an $\mathbb{F}_{q} G_{1}$-module;

$6 A:=\operatorname{ActionGroup}\left(M_{1}\right) ; \theta:=\operatorname{Action}\left(M_{1}\right)$;

(* Note that $\operatorname{Domain}(\theta)=G_{1} *$ )

$7 \quad[\psi]^{(m)}:=\operatorname{NormalSeriesmaps}(A) ;$

8 for $i \in[1 \ldots r]$

11 for $i \in[1 \ldots m]$

$$
\begin{aligned}
& \text { do Choose } e_{i} \in G \text { with } V_{i}^{\rho\left(e_{i}\right)}=V_{1} \text {; } \\
& \quad \text { Let } \alpha_{e_{i}}: G \rightarrow G \text { be the map } \alpha_{e_{i}}(g)=e_{i}^{-1} g e_{i} \text {; }
\end{aligned}
$$$$
\left(* \text { So } \alpha_{e_{i}}\left(G_{V_{i}}\right)=G_{1} \text { and } \operatorname{Domain}\left(\theta \circ \alpha_{e_{i}}\right)=G_{V_{i}} *\right)
$$

$12 \operatorname{do} \zeta_{i}:=\psi_{i} \circ \theta \circ \alpha_{e_{1}} \times \cdots \times \psi_{i} \circ \theta \circ \alpha_{e_{r}}$;

13 return $[\tau]^{(k)}$ cat $[\zeta]^{(m)}$;

Groups in Class C3 will be subdivided into those that act absolutely irreducibly and those that do not. Absolutely irreducible subgroups of $\mathrm{GL}(n, q)$ in Class C3 that are isomorphic to subgroups of $\Gamma \mathrm{L}\left(n / e, q^{e}\right)$ containing a field automorphism of order $e$ become imprimitive with $e$ blocks of imprimitivity when regarded as 
subgroups of $\operatorname{GL}\left(n, q^{e}\right)$, so our method in that case is to extend the ground field of $G$ and call ImprimitiveMAPs. Groups that are not absolutely irreducible are handled by the following procedure.

\section{AbsolutelyReduciblemaps $(G)$}

Assume: $G$ acts irreducibly but not absolutely irreducibly on $M_{G}$.

1 Construct (using the methods outlined in [20]) an isomorphism $\psi: G \rightarrow H \leqslant \mathrm{GL}\left(\frac{n}{e}, q^{e}\right)$ where $H$ is the irreducible reduced-degree representation of $G$;

$2[\phi]^{(k)}:=\operatorname{Normal} \operatorname{SeriesMaps}(H) ;$

3 return $\operatorname{PULlBACK}\left([\phi]^{(k)}, \psi\right)$;

For groups in Class C4, which preserve a tensor product decomposition $V_{G}=$ $U \otimes V$, the induced actions of $G$ on $U$ and $V$ are only projective actions. In order to use the pullback construction, we therefore require that the preliminary sequence computed on the images of these actions are correct on scalars. Recall that we are claiming that the procedures for processing groups in Classes C4-C9 return preliminary sequences that are correct on scalars, and that we are assuming by induction that this is true for recursive calls of these procedures. So we need to assume that the induced actions of $G$ on $U$ and $V$ do not lie in any of the classes $\mathrm{C} 1, \mathrm{C} 2, \mathrm{C} 3$. To justify this, we shall now show that if either of the actions were in $\mathrm{C} 1, \mathrm{C} 2$ or $\mathrm{C} 3$ then so would $G$ be, in which case we would have already called one of the earlier procedures on $G$.

TheOREM 2.2. Let $G$ preserve a tensor decomposition of $V_{G}$, as $V_{G}=U \otimes W$.

1. If $G$ acts reducibly on $U$ then $G$ acts reducibly on $V_{G}$.

2. If $G$ acts imprimitively on $U$ then $G$ acts imprimitively on $V_{G}$.

3. If the action of $G$ on $U$ is not absolutely irreducible then $G$ is not absolutely irreducible on $V_{G}$.

4. If the action of $G$ on $U$ is semilinear then $G$ is semilinear on $V_{G}$.

Proof. The proofs of 1 and 2 are straightforward. For 3, assume that the action of $G$ on $U$ is not absolutely irreducible. Then there exists a non-scalar $C \in \mathrm{GL}(U)$ that centralises the action of $g$ on $U$ for all $g \in G$. Now let $D$ be a matrix in $\operatorname{GL}(n, q)$ that preserves the decomposition $U \otimes W$ of $V_{G}$ and acts as $C$ on $U$ and as $I_{W}$ on $W$. Then $D$ is a non-scalar matrix that centralises $G$.

For 4 , we know from 3 that $G$ has a normal irreducible subgroup $N$ that does not act absolutely irreducibly. Let $D$ be the non-scalar matrix that centralises $N$ and acts as $I_{W}$ on $W$ as constructed above. Let $g \in G$, let $g_{1}$ be the induced action of $g$ on $U$ and let $C$ be the induced action of $D$ on $U$. Then since $G$ is semilinear on $U$ there exists $i=i(g)$ such that $C g_{1}=g_{1} C^{q^{i}}$, but the action of $D$ on $W$ is trivial so $D g=g D^{q^{i}}$ and $G$ is semilinear. 


\section{TensorMaps $(G)$}

Assume: $G$ lies in Class C4 but not in Classes C1, C2, C3.

(* We use the algorithm of $[\mathbf{2 4}]$ to find $U$ and $W$ with $V_{G}=U \otimes W *$ )

$1 \quad \psi_{1}:=\operatorname{ProjectiveAction}(U) ; \psi_{2}:=\operatorname{ProjectiveAction}(W)$;

$2 \quad H_{1}:=\operatorname{im}\left(\psi_{1}\right) ; H_{2}:=\operatorname{im}\left(\psi_{2}\right)$;

$3[\alpha]^{\left(k_{1}\right)}:=\operatorname{NormalSeriesMaps}\left(H_{1}\right) ;[\beta]^{\left(k_{2}\right)}:=\operatorname{Normal} \operatorname{Seriesmaps}\left(H_{2}\right)$;

(* By Theorem 2.2 and induction, $[\alpha]^{\left(k_{1}\right)}$ and $[\beta]^{\left(k_{2}\right)}$ are correct on scalars $*$ )

$4[\phi]^{\left(k_{1}+k_{2}-1\right)}:=\operatorname{Pullback}\left([\alpha]^{\left(k_{1}-1\right)}, \psi_{1}\right)$ cat Pullback $\left([\beta]^{\left(k_{2}-1\right)}, \psi_{2}\right)$

cat $\operatorname{SCALARMAP}(n, q)$;

5 return $[\phi]^{\left(k_{1}+k_{2}-1\right)}$;

Groups in Class C5 are conjugates of subgroups of $\langle\mathrm{GL}(n, \mathbb{K}), Z\rangle$, where $Z$ is the scalar subgroup of $\operatorname{GL}(n, q)$ and $\mathbb{K}$ is a proper subfield of $\mathbb{F}_{q}$. They therefore give rise to a projective homomorphism $\psi: G \rightarrow H \leqslant \mathrm{GL}(n, \mathbb{K})$ with kernel $Z_{G}$.

It is easy to see that if the image $H$ of $\psi$ is reducible or imprimitive then so is $G$. Furthermore, we leave it to the reader to prove that if $H$ is reducible but not absolutely irreducible, then $G$ is either reducible or is not absolutely irreducible, and if $H$ is absolutely irreducible but semilinear, then $G$ is either semilinear or imprimitive. So we may assume that $H$ is not in Class $\mathrm{C} 1, \mathrm{C} 2$ or $\mathrm{C} 3$, and hence that the preliminary sequence computed for $H$ is correct on scalars.

\section{SmallerFieldMaps $(G)$}

Assume: $G$ lies in Class C5 but not in Classes C1, C2, C3.

1 Construct (using the methods given in [14]) a projective homomorphism $\psi: G \rightarrow H \leqslant \mathrm{GL}(n, \mathbb{K})$ for a proper subfield $\mathbb{K}$ of $\mathbb{F}_{q} ;$

$2[\phi]^{(k)}:=\operatorname{Normal} \operatorname{SeriesMaps}(H) ;$

$\left(*[\phi]^{(k)}\right.$ is correct on scalars $\left.*\right)$

$3[\alpha]^{(k-1)}:=\operatorname{PullBACK}\left([\phi]^{(k-1)}, \psi\right)$;

4 return $[\alpha]^{(k-1)}$ cat $\operatorname{SCALARMAP}(n, q)$;

Groups $G$ in Class C6 have a normal subgroup $E$ that is either extraspecial of order $r^{2 m+1}$ or a 2-group of symplectic type of order $2^{2 m+2}$ for a prime $r$ and integer $m \geqslant 1$, where $|Z(E)|=r$ or 4 , respectively. (A 2-group of symplectic type is a central product of an extraspecial 2-group with a cyclic group of order 4.) So we have an action of $G / E$ on $E / Z(E)$ giving rise to an embedding $\psi: G / E \rightarrow \operatorname{GL}(2 m, r)$. We want to apply NormalSERIESMAPs recursively to $\operatorname{im}(\psi)$ and then to pull the resulting preliminary series back through $\psi$ and, for this to work properly, we need to be able to assume that $\operatorname{im}(\psi)$ is irreducible, thereby ensuring that $O_{r}(\operatorname{im}(\psi))=1$. In the next few lemmas we show that, if $\operatorname{im}(\psi)$ is reducible, then $G$ lies in one of the classes C2, C3, C4, which we can assume is not the case. We shall prove the lemmas only for the extraspecial case.

LEMMA 2.3. Let $r$ be a prime and let $E \leqslant \mathrm{GL}\left(r^{m}, q\right)$ be an extraspecial $r$-group of order $r^{2 m+1}$, with $r \nmid q$. Then $E$ is the central product of $m$ extraspecial groups of order $r^{3}$. Furthermore, $E$ preserves a tensor decomposition of $M_{E}$ as $M_{E}=$ $M_{1} \otimes \cdots \otimes M_{m}$, where the restriction of $E$ to each $M_{i}$ is a representation of an extraspecial group of order $r^{3}$.

Proof. See [15, Theorem 5.5.5]. 
LEMmA 2.4. Let $E \leqslant \mathrm{GL}\left(2^{m}, q\right)$ be a 2-group of symplectic type of order $2^{2 m+2}$, with $q \equiv 1(\bmod 4)$. Then $E$ preserves a tensor decomposition of $M_{E}$ as $M_{E}=$ $M_{1} \otimes \cdots \otimes M_{m}$.

LEMma 2.5. Let $r$ be a prime and let $E \leqslant \mathrm{GL}\left(r^{m}, q\right)$ be an extraspecial $r$-group of order $r^{2 m+1}$ or a 2-group of symplectic type of order $2^{2 m+2}$. Let $H$ be a proper subgroup of $E$ that contains $Z(E)$. Then $H$ acts reducibly.

Proof. It is enough to show $H$ acts reducibly when $|G: H|=r$. Assume first that $m=1$, so $|E|=r^{3}$. Then $H$ has order $r^{2}$, so is abelian. By [15, Theorem 3.2.3], $H$ has no faithful irreducible representations, so $H$ must act reducibly.

In general $E$ is a central product of $m$ extraspecial groups of order $r^{3}$. As $H$ has index $r$ in $E$, it can be seen that $H$ is a central product of $r-1$ extraspecial groups of order $r^{3}$ and an abelian group of order $r^{2}$. Hence one of the tensor factors of the representation of $H$ in $\operatorname{GL}\left(r^{m}, q\right)$ is reducible and $H$ acts reducibly by Theorem 2.2 .

THEOREM 2.6. Let $G \leqslant \operatorname{GL}\left(r^{m}, q\right)$ act irreducibly and have a normal subgroup $E$ that is either an extraspecial $r$-group or, when $r=2$, a 2-group of symplectic type. Let $L$ be the $\mathbb{F}_{r} G$-module defined by the conjugation action of $G$ on $E / Z(E)$. If $G$ acts reducibly on $L$ then either $G$ acts imprimitively on $V_{G}$, or $G$ acts as a group of semilinear automorphisms on $V_{G}$, or $G$ preserves a tensor decomposition of $V_{G}$.

Proof. This follows from the general theory of Smash described in [18]. Briefly, Clifford's Theorem [15, Theorem 3.4.1] states that, if $N \unlhd G$, then $V_{G}$ splits as a direct sum $W_{1} \oplus \cdots \oplus W_{k}$ of irreducible $\mathbb{F}_{q} N$-modules, all of the same dimension. For some $t, s \geqslant 1$, with $t s=k$, the $W_{i}$ 's partition into $t$ sets containing $s$ pairwise isomorphic $\mathbb{F}_{q} N$-modules each, and if $V_{1}, \ldots, V_{t}$ are each the sum of pairwise isomorphic $W_{i}$, so that $V=V_{1} \oplus \cdots \oplus V_{t}$, then $G$ permutes the $V_{i}$ transitively.

If $G$ acts reducibly on $L$ then $G$ normalises some proper subgroup, $N$ say, of $E$. By the previous lemmas, $N$ acts reducibly on $V_{G}$ so, in the context of Clifford's Theorem, we must have $k>1$.

If $t>1$, then $\left\{V_{1}, \ldots, V_{t}\right\}$ forms a block system for $G$ and $G$ is imprimitive. Otherwise $V_{G}$ decomposes as a direct sum of $k$ irreducible pairwise isomorphic $\mathbb{F}_{q} N$-modules, $W=W_{1}, W_{2}, \ldots, W_{k}$. From the descriptions given in [18] we see that if each $W_{i}$ acts absolutely irreducibly then $G$ preserves a tensor decomposition of $V_{G}$ as $U \otimes W$, where $N$ acts as scalars on $U$. If each $W_{i}$ does not act absolutely irreducibly then $G$ is semilinear.

Let $G \leqslant \mathrm{GL}\left(r^{m}, q\right)$ act irreducibly, and suppose that $G$ has an extraspecial normal $r$-subgroup or a normal 2 -subgroup of symplectic type $E$. Then methods described in $[\mathbf{1 8}]$ provide us with the action of $G$ on the $\mathbb{F}_{r}$ G-module $E / Z(E)$, where $Z(E)$ is a group of scalar matrices of order $r$, or of order 4 if $E$ is a 2group of symplectic type. The kernel of this action is $E Z_{G}$. We also obtain elements $e_{1}, \ldots, e_{2 m} \in E$ whose images in $E / Z(E)$ are a free basis for the elementary abelian group. We wish to construct a homomorphism $\alpha: E Z_{G} \rightarrow E / Z(E)$ that has kernel $Z_{G}$. 
TheOREM 2.7. Let $E$ and $e_{1}, \ldots, e_{2 m}$ be as defined above and let $\bar{e}_{1}, \ldots, \bar{e}_{2 m}$ be the images of the $e_{i}$ in $E / Z(E)$. Let $z$ be a scalar element of $G$ of order $r$. Define $\alpha: E Z_{G} \rightarrow E / Z(E)$ by $\alpha(g)=\bar{e}_{1}^{i_{1}} \cdots \bar{e}_{2 m}^{i_{2 m}}$, where $\left[g, e_{j}\right]=z^{i_{j}}$ for $1 \leqslant j \leqslant 2 m$. Then $\alpha$ is a homomorphism with kernel $Z_{G}$.

Proof. If $j \in\{1, \ldots, 2 m\}$ and $g \in E Z_{G}$, then $\left[g, e_{j}\right] \in Z_{E}$, since $e_{i}$ and $g$ commute modulo $Z_{G}$ and $\left[g y, e_{j}\right]=\left[g, e_{j}\right]$ for any scalar $y$. Also, $\left[g h, e_{j}\right]=\left[g, e_{j}\right]^{h}\left[h, e_{j}\right]=$ $\left[g, e_{j}\right]\left[h, e_{j}\right]$ for any $g, h \in E Z_{G}$ and $j \in\{1, \ldots, 2 m\}$, so $\alpha$ is a homomorphism. Finally, $\left[g, e_{j}\right]=1$ for all $1 \leqslant j \leqslant 2 m$ if and only if $g \in Z\left(E Z_{G}\right)=Z_{G}$, so $\operatorname{ker}(\alpha)=Z_{G}$ and $\alpha$ is surjective.

\section{ExtraSpecialNormaliserMaps $(G)$}

Assume: $G$ lies in Class C6 but not in Classes C1, C2, C3, C4.

(* We find $E$ and the module $L:=E / Z(E)$ as described in $[\mathbf{1 8}] *$ )

$1 \psi:=\operatorname{Action}(L) ; H:=\operatorname{ActionGroup}(L)$;

$2[\phi]^{(k)}:=\operatorname{Normal} \operatorname{SeriesMaps}(H) ;$

$\left(* \operatorname{ker}\left(\phi_{k}\right)=O_{r}\left(G / E Z_{G}\right)=1\right.$ by Theorem $\left.2.6 *\right)$

3 Let $\alpha: E Z_{G} \rightarrow E / Z(E)$ be the homomorphism defined in Theorem 2.7.

4 return Pullback $\left([\phi]^{(k)}, \psi\right)$ cat $\alpha$ cat $\operatorname{Scalarmap}(n, q)$;

We require results for the tensor induced case similar to those in the tensor case.

TheOREM 2.8. Let $G$ be a tensor induced group. So $V_{G}=V_{1} \otimes \cdots \otimes V_{m}$. For $1 \leqslant i \leqslant m$ let $G_{i}$ be the subgroup of $G$ that stabilises $V_{i}$ and let $A_{i}$ be the restriction of $G_{i}$ to $V_{i}$. Note that the $A_{i}$ are all isomorphic.

1. If $A_{1}$ acts reducibly on $V_{1}$ then $G$ acts reducibly on $V_{G}$.

2. If $A_{1}$ acts imprimitively on $V_{1}$ then $G$ acts imprimitively on $V_{G}$.

3. If the action of $A_{1}$ on $V_{1}$ is not absolutely irreducible then $G$ acts reducibly on $V_{G}$.

4. If the action of $A_{1}$ on $V_{1}$ is semilinear then $G$ acts imprimitively on $V_{G}$.

Proof. The proofs of 1 and 2 are straightforward. For 3 , if $\mathbb{F}_{q^{e}}$ is the splitting field for $V_{1}$ under $A_{1}$, then $\mathbb{F}_{q^{e}}$ is the splitting field for $V_{i}$ under $A_{i}$ for $1 \leqslant i \leqslant m$. Over $\mathbb{F}_{q^{e}}$ we have $V_{i}=L_{i} \oplus L_{i}^{\sigma} \oplus \cdots \oplus L_{i}^{\sigma^{e-1}}$, where $L_{i}$ is absolutely irreducible and $\sigma$ is the field automorphism. Then the subspace

$$
\left(L_{1} \otimes \cdots \otimes L_{m}\right) \oplus\left(L_{1}^{\sigma} \otimes \cdots \otimes L_{m}^{\sigma}\right) \oplus \cdots \oplus\left(L_{1}^{\sigma^{e-1}} \otimes \cdots \otimes L_{m}^{\sigma^{e-1}}\right)
$$

is fixed by the field automorphism $\sigma$. Hence it can be written over $\mathbb{F}_{q}$. It is also fixed by all permutations of the tensor factors, hence by elements of $G$. So $G$ is reducible. The proof of 4 is similar and is left to the reader.

The code for TEnsorInducedMaps is similar to that for ImPrimitiveMaPs. 


\section{TensorInducedMaps $(G)$}

Assume: $G$ lies in Class C7, but not in Classes C1, C2, C3, C4.

1 Construct (using methods described in [25]) a set of tensor factors $\Omega=\left\{V_{1}, \ldots, V_{r}\right\}$ permuted by $G$, and a homomorphism $\rho: G \rightarrow \operatorname{Sym}(r) ;$

$2 \quad \Sigma:=\operatorname{im}(\rho)$;

$3[\tau]^{(k)}:=\operatorname{Pullback}(\operatorname{NormalSeriesmaps}(\Sigma), \rho)$;

$4 G_{1}:=G_{V_{1}}$, the stabiliser of $V_{1}$ in $G$;

5 Let $M_{1}$ be $V_{1}$ considered as an $\mathbb{F}_{q} G_{1}$-module;

$6 \quad \theta:=\operatorname{ProjectiveAction}\left(V_{1}\right) ; A:=\operatorname{im}(\theta) ;$

$7[\psi]^{(m)}=\operatorname{Normal} \operatorname{SeriesMaps}(A) ;$

$\left(*[\psi]^{(m)}\right.$ is correct on scalars by Theorem 2.8 and induction $*$ )

8 for $i \in[1 \ldots r]$

for $i \in[1 \ldots m-1]$

$$
\text { do Choose } e_{i} \in G \text { with } V_{i}^{\rho\left(e_{i}\right)}=V_{1} \text {; }
$$

$$
\text { do } \zeta_{i}:=\psi_{i} \circ \theta \circ \alpha_{e_{1}} \times \cdots \times \psi_{i} \circ \theta \circ \alpha_{e_{r}} ;
$$

$\left(* Z_{A}=\operatorname{ker}\left(\psi_{m-1}\right)\right.$ and the $\zeta_{i}$ are normal homomorphisms $*$ )

\section{3 return $[\tau]^{(k)}$ cat $[\zeta]^{(m-1)}$ cat $\operatorname{ScAlaRMAP}(n, q)$;}

If $G$ is in Class C8 then the algorithms of [28] will return the type of classical group normalised by $G$ and, if $G$ does not contain $\operatorname{SL}(n, q)$, they will also return a classical form $\Phi$ that is fixed by $G$ modulo scalars. We denote the action of $g \in G$ on the form $\Phi$ by $\Phi^{g}$. So, for $g \in G, \Phi^{g}=w \Phi$ for some non-zero scalar $w \in \mathbb{F}_{q}$.

The code for handling these groups is complicated by the fact that the structure of the normaliser $\Delta$ of the quasisimple classical group $\Omega$ in $\operatorname{GL}(n, q)$ varies from case to case, and also by our need to push the scalar subgroup $Z_{G}$ of $G$ to the bottom of the normal series. We therefore present separate code for the four cases.

Here is a brief summary of the structure of $\Delta$; we refer the reader to $[\mathbf{2 2}$, Chapter 2] for further details. There are subgroups $S$ and $I$ of $\Delta$ with $\Omega \leqslant S \leqslant I \leqslant \Delta$. In the linear case, $I=\Delta=\mathrm{GL}(n, q)$. Otherwise, $\Delta$ (sometimes known as the conformal unitary, symplectic or orthogonal group) is the subgroup of $\operatorname{GL}(n, q)$ that fixes the form modulo scalars, and $I$ is the subgroup that fixes the form; that is, $\Phi^{g}=\Phi$ for $g \in I$. In the unitary groups and in all groups in even characteristic or odd degree, $\Delta$ is generated by $I$ and scalar matrices, but in the symplectic and orthogonal groups in odd characteristic and even degree, the subgroup generated by $I$ and the scalars has index two in $\Delta$. In all cases, $S$ is the 'special' subgroup of matrices of determinant 1 . In the symplectic case $S=I$, and in the orthogonal groups $|I: S|=1$ or 2 , respectively, in even and odd characteristics. The quasisimple group $\Omega$ is the derived subgroup of $S$ and has index 2 in $S$ in the orthogonal groups, and is equal to $S$ otherwise.

Membership of elements $g$ of $\Delta$ in $I$ and in $S$ is readily tested by computing $\Phi^{g}$ and $\operatorname{det}(g)$. Membership of elements $g$ of $S=\mathrm{SO}^{\epsilon}(n, q)$ in $\Omega=\Omega^{\epsilon}(n, q)$ can also be easily computed using the function SPINORNorm; see [33, p. 163] for a description. The value of $\operatorname{SpinorNorm}(g)$ is 0 when $g \in \Omega$ and 1 otherwise. It turns out that, if $n$ is even, $q$ is odd and $\operatorname{det}(\Phi)$ is a non-square, then $-I_{n}$ has spinor norm 1 , and so $S$ and $\Omega$ are equal mod scalars, and we take the map $\phi_{3}$ in $\operatorname{C8MapsOrTh}(G)$ to be trivial. In all other cases, $-I_{n}$ has spinor norm 0 and $\left|\operatorname{PSO}^{\epsilon}(n, q): \mathrm{P}^{\epsilon}(n, q)\right|=2$. Note that $\phi_{3}$ is well-defined in this situation, because the scalar $z_{g}$ is unique up to multiplication by $-I_{n}$. 
Let $\gamma: \mathbb{F}_{q}^{\times} \rightarrow \mathbb{F}_{q}^{\times} / N$ with $N:=\left\langle\operatorname{det}(g) \mid g \in Z_{G}\right\rangle$ be the natural epimorphism with the image represented as a group with polycyclic presentation. We denote by $\operatorname{det}_{Z}$ the homomorphism $\gamma \circ$ det $: \operatorname{GL}(n, q) \rightarrow \mathbb{F}_{q}^{\times} / N$. Note that, with $I$ and $S$ defined as above, we have $\operatorname{ker}\left(\operatorname{det}_{Z}\right) \cap I=S Z_{G}$. Note also that the scalar $z_{g}$ in the procedures below, which is chosen to make $g z_{g}^{-1} \in S$ in all cases, can be easily computed from $\operatorname{det}(g)$ and $\Phi^{g}$.

\section{$\operatorname{C8MapsSL}(G)$}

Assume: $\mathrm{SL}(n, q) \leqslant G \leqslant \mathrm{GL}(n, q)$

$1 \quad \phi_{1}:=\operatorname{det}_{Z} ;$

2 Define $\phi_{2}:\left\langle\operatorname{SL}(n, q), Z_{G}\right\rangle \rightarrow \operatorname{PSL}(n, q)$ from the projective homomorphism $g \mapsto g z_{g}^{-1}$, where $z_{g}$ is a scalar with $\operatorname{det}\left(z_{g}\right)=\operatorname{det}(g)$;

3 return $\left(\phi_{1}, \phi_{2}, \operatorname{SCAlARMAP}(n, q)\right)$;

\section{C8MapsUnitary $(G)$}

Assume: $G$ contains and normalises $\mathrm{SU}(n, \sqrt{q})$.

(* Let $\Phi$ be the unitary form associated to $G *$ )

$1 \quad \phi_{1}:=\operatorname{det}_{Z}$;

2 Define $\phi_{2}:\left\langle\mathrm{SU}(n, q), Z_{G}\right\rangle \rightarrow \operatorname{PSU}(n, \sqrt{q})$ from the projective homomorphism $g \mapsto g z_{g}^{-1}$, where $z_{g}$ is a scalar with $\operatorname{det}\left(z_{g}\right)=\operatorname{det}(g)$ and $\Phi^{g}=z_{g}^{\sqrt{q}+1} \Phi$;

3 return $\left(\phi_{1}, \phi_{2}, \operatorname{SCALARMAP}(n, q)\right)$;

\section{$\mathrm{C} 8 \mathrm{MapsSp}(G)$}

Assume: $G$ contains and normalises $\operatorname{Sp}(n, q)$.

(* Let $\Phi$ be the symplectic form associated to $G *$ )

1 Define $\phi_{1}: G \rightarrow \mathbb{Z}_{2}=\{0,1\}$ as follows:

$2 \quad$ Let $\omega \in \mathbb{F}_{q}$ be such that $\Phi^{g}=\omega \Phi$;

3 if $\omega$ is a square then $\phi_{1}(g):=0$ else $\phi_{1}(g):=1$;

$\left(* \phi_{1}\right.$ is always trivial when $q$ is even $*$ )

4 Define $\phi_{2}:\left\langle\operatorname{Sp}(n, q), Z_{G}\right\rangle \rightarrow \operatorname{PSp}(n, q)$ from the projective homomorphism $g \mapsto g z_{g}^{-1}$, where $z_{g}$ is a scalar with $\Phi^{g}=z_{g}^{2} \Phi$

5 return $\left(\phi_{1}, \phi_{2}, \operatorname{SCALARMAP}(n, q)\right)$;

\section{C8MapsOrth $(G)$}

Assume: $G$ contains and normalises $\Omega^{\epsilon}(n, q)$.

(* Let $\Phi$ be the orthogonal or quadratic form associated to $G *$ )

1 Define $\phi_{1}: G \rightarrow \mathbb{Z}_{2}=\{0,1\}$ as follows:

$2 \quad$ Let $\omega \in \mathbb{F}_{q}$ be such that $\Phi^{g}=\omega \Phi$;

3 if $\omega$ is a square then $\phi_{1}(g):=0$ else $\phi_{1}(g):=1$;

(* $\phi_{1}$ is always trivial when $q$ is even or $n$ is odd $*$ )

$4 \phi_{2}:=\operatorname{det}_{Z}$;

5 Define $\phi_{3}:\left\langle\mathrm{SO}^{\epsilon}(n, q), Z_{G}\right\rangle \rightarrow \mathbb{Z}_{2}=\{0,1\}$ as follows:

6 if $n$ is even, $q$ is odd, and $\operatorname{det}(\Phi)$ is a non-square

$7 \quad$ then $\phi_{3}(g)=0$

$8 \quad$ else $\phi_{3}(g):=\operatorname{SPINORNORM}\left(g z_{g}^{-1}\right)$

where $z_{g}$ is a scalar with $\operatorname{det}\left(z_{g}\right)=\operatorname{det}(g)$ and $\Phi^{g}=z_{g}^{2} \Phi$;

9 Define $\phi_{4}:\left\langle\Omega^{\epsilon}(n, q), Z_{G}\right\rangle \rightarrow \mathrm{P} \Omega^{\epsilon}(n, q)$ from the projective homomorphism $g \mapsto g z_{g}^{-1}$, where $z_{g}$ is a scalar with $\operatorname{det}\left(z_{g}\right)=\operatorname{det}(g)$ and $\Phi^{g}=z_{g}^{2} \Phi$;

10 return $\left(\phi_{1}, \phi_{2}, \phi_{3}, \phi_{4}, \operatorname{ScAlarMaP}(n, q)\right)$; 
To deal with groups in Class C9, we make use of the following procedure, developed by Leedham-Green and O'Brien in [25], to perform membership testing in a normal subgroup of a group.

\section{$\operatorname{ElementinN}(G, N, g)$}

Input: Group $G$, normal subgroup $N$ of $G$, element $g \in G$.

$1 m:=|g|$;

2 for some number $I$ of times

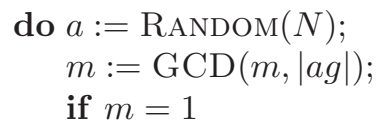

\section{then return true;}

\section{return false;}

It is easy to see that if ELEMENTINN returns true then $g \in N$, but it could conceivably return a false negative; that is, it could claim that an element $g \in N$ is not in $N$. We shall only be applying it when $N$ is quasisimple, in which case an unpublished result of Babai, Pálfy and Saxl enables us to prove an upper bound on the probability of a false negative as a function of the chosen number $I$ of iterations of the loop of the procedure. In other words, we can make ELEMENTINN into a Monte-Carlo algorithm in this situation. We omit the details, which can be found in $[4]$.

Using ElementinN, we can test whether a group $G$ is perfect. The commutator subgroup of $G$ is equal to the normal closure of the subgroup $H$ generated by the commutators of the generators of $G$. Using a variant of the product replacement algorithm we can compute random elements of the normal closure of a subgroup $H$ of $G$. Therefore, using a variant of $\operatorname{ELEmEnTINN}$ where $\operatorname{RAndom}(N)$ in Line 3 is replaced by a function computing a random element of the normal closure of $N$, we can determine if each generator of $G$ lies in the normal closure of $H$. Once again, this method can return false negatives but not false positives and, if the derived group of $G$ is quasisimple, then we can estimate upper bounds for the probability of a false negative.

This in turn enables us to define a procedure StableDerivative, which will compute the last member $G^{(\infty)}$ of the derived series of a group $G$ and, if $G^{(\infty)}$ is quasisimple, then the probability of an incorrect result can be estimated and made as low as we wish.

Finally, given a normal subgroup $N$ of $G$ for which the index $|G: N|$ is small, we can use ElEMENTINN to enumerate the cosets of $N$ in $G$, and then construct the permutation representation $\operatorname{Coset} \operatorname{Image}(\mathrm{G}, \mathrm{N})$ of $G$ on the right cosets of $N$ in $G$.

We are now able to present the algorithm AlmostSimplemaps. Notice that we apply $\operatorname{Cosetimage}(\mathrm{G}, \mathrm{N})$ only when $N$ is quasisimple, and we apply StableDerivative $(\mathrm{G})$ only when $G^{(\infty)}$ is quasisimple, so we can choose parameters to make the probability of a false result as small as we please. Note also that in the application of $\operatorname{Coset} \operatorname{Image}(\mathrm{G}, \mathrm{N}), G / N$ is contained in the outer automorphism group of a finite simple group, and so will be moderately small.

If we did make an error and underestimate the group $N$ at this stage, then the error would be detected when we carried out constructive recognition of the simple group, since we would then be able to carry out membership testing in $N$ 
deterministically, and we could verify that our coset representatives of $N$ in $G$ were all genuinely in distinct cosets.

\section{AlmostSimplemaps $(G)$}

Assume: $G$ is absolutely irreducible and almost simple modulo scalars.

$1 \hat{G}:=\langle G, z\rangle$ where $z$ is a generator of $Z_{\mathrm{GL}(n, q)}$;

(* We actually compute a preliminary sequence for $\hat{G} *$ )

$2 \quad N:=\operatorname{StableDerivative}(G)$;

$3 \hat{N}:=\langle N, z\rangle$;

$4 \phi_{1}:=\operatorname{Cosetimage}(\hat{G}, \hat{N})$;

5 Let $\phi_{2}: \hat{N} \rightarrow N / Z(N)$ be defined by the projective homomorphism $\hat{N} \rightarrow N$ which maps $g \mapsto z g$, where $z$ is a scalar with $\operatorname{ElEment} \operatorname{InN}(\hat{N}, N, z g)=$ true;

6 return $\left(\phi_{1}, \phi_{2}, \operatorname{ScAlARMAP}(n, q)\right)$;

During the computations that we carry out when applying NormALSERIESMAPS to a group $G \leqslant \mathrm{GL}(n, q)$, we can compute a multiset, $M=\left\{S_{1}^{e_{1}}, \ldots, S_{m}^{e_{m}}, \mathbb{Z}_{p_{1}}^{t_{1}}, \ldots\right.$, $\left.\mathbb{Z}_{p_{r}}^{t_{r}}\right\}$, where each $S_{i}$ is a nonabelian simple group, and the multiset of composition factors of $G / O_{p}(G)$ is a subset of $M$. We only require $M$ to contain the names of the simple groups $S_{i}$, and not any representations of the groups themselves. We shall use this multiset $M$ in Subsection 2.3 to obtain a probability estimate of the number of generators required to generate the subgroups in a chief series of $G$.

We may compute $M$ as follows. We assume that there is no difficulty in identifying the composition factors that arise from permutation groups. The only algorithms above that do not use recursion are AlmostSimpleMAPs and the four C8MAPS procedures. At each step of these algorithms we store either the factorisation of the order of the soluble image or the standard name of the quasisimple image, which (in the case of AlmostSimpleMAPs), we can find using non-constructive recognition. Hence, by recursion, with all maps $\phi_{i}$ constructed by NormaLSERIESMAPS we may store either a factorization of $\operatorname{im}\left(\phi_{i}\right)$ if it is soluble, or the standard name of one the isomorphic simple groups in $\operatorname{im}\left(\phi_{i}\right)$ along with an upper bound for the number of copies. Observe also that this provides us with a test for the solubility of a matrix group.

\subsection{Evaluating the Images and Kernels}

We assume now that we have successfully computed a preliminary sequence $[\phi]^{(k)}$ for $G \leqslant \mathrm{GL}(n, q)$. At this stage we know only a superset of the composition factors of $G$. The next step is to compute the images and kernels of the $\phi_{i}$, which we do as follows.

Let $G_{0}=G$. For $i=1, \ldots, k$, do the following.

1. Compute generators of the image $Q_{i}$ of the restriction of $\phi_{i}$ to $G_{i-1}$ by applying $\phi_{i}$ to a set of generators of $G_{i-1}$. (For $i=0$ we just use the given generating set of $G$.)

2. If $Q_{i}$ is insoluble then identify $Q_{i}$ constructively (see Subsection 1.1).

3. If $i<k$ then compute generators of a subgroup $G_{i}$ of $\operatorname{ker}\left(\phi_{i}\right) \cap G_{i-1}$, for which we hope that $G_{i} O_{p}(G)=\left(\operatorname{ker}\left(\phi_{i}\right) \cap G_{i-1}\right) O_{p}(G)$.

Since the $\phi_{i}$ are directly computable homomorphisms, Step 1 is straightforward, and we shall discuss Steps 2 and 3 shortly. In the next subsection, we shall describe the process in which we verify the correctness of $G_{i} O_{p}(G)=\left(\operatorname{ker}\left(\phi_{i}\right) \cap G_{i-1}\right) O_{p}(G)$ for 
$0<i<k$. After this verification we know that $G_{i} O_{p}(G)=K_{i}$ for $0 \leqslant i<k$, where $K_{i}$ are the subgroups associated with the preliminary sequence (Definition 2.1). Note that, from the construction of $[\phi]^{(k)}$, we know that $\operatorname{ker}\left(\phi_{k}\right)=O_{p}(G)$, but we do not attempt to find generators of $O_{p}(G)$ at this stage. We shall do that later, in Subsection 2.5.

We turn now to the constructive recognition of the insoluble images $Q_{i}$ in Step 2 . Assume that $\operatorname{im}\left(\phi_{i}\right)$ is a direct product $S^{l}$ of isomorphic nonabelian simple groups. Since (assuming that the kernels calculated so far are correct modulo $O_{p}(G)$ ) $Q_{i}=$ $\phi_{i}\left(G_{i-1}\right)$ is a normal subgroup of $\operatorname{im}\left(\phi_{i}\right), Q_{i}$ is a direct product $S^{m}$ of some possibly smaller number $m$ of copies of $S$ ( $Q_{i}$ could even be trivial). Furthermore, in some cases, particularly when $\phi_{i}$ arises from an application of IMPRIMITIVEMAPS or TEnsorInduCEDMAPS, $\operatorname{im}\left(\phi_{i}\right)$ is defined as a subdirect product of a possibly larger direct product of copies of $S$. So we must first determine $l$ and then $m$.

Subdirect products of direct products of isomorphic nonabelian simple groups are described by the following result, of which we leave the proof to the reader.

Proposition 2.9. Let $G=S_{1} \times S_{2} \times \cdots \times S_{n}$, where the $S_{i}$ are all isomorphic to the same nonabelian simple group $S$, and let $H$ be a subdirect product of $G$. Then there is a partition $\mathcal{P}_{i}(1 \leqslant i \leqslant l)$ of the set $\{1,2, \ldots, n\}$ such that $H \cong S^{l}$, and the intersection of $H$ with each of the groups $G_{i}:=\times_{j \in \mathcal{P}_{i}} S_{j}$ for $1 \leqslant i \leqslant l$ is a diagonal subgroup of $G_{i}$ isomorphic to $S$.

In practice, provided that we can compute the projections of $G$ onto the $S_{i}$ and orders of elements in $G$, it is easy to calculate the partition $\mathcal{P}_{i}$ with an arbitrarily small probability of error. We choose random elements $g=\left(g_{1}, \ldots, g_{n}\right)$ of $G$ and compute the orders of the components $g_{i}$. Then $\left|g_{j}\right|=\left|g_{k}\right|$ whenever $j$ and $k$ are in the same set $\mathcal{P}_{i}$ but, with probability greater than $1 / 2,\left|g_{j}\right| \neq\left|g_{k}\right|$ when $j$ and $k$ are in different sets $\mathcal{P}_{i}$.

So we can identify $\operatorname{im}\left(\phi_{i}\right)$ and then $\phi_{i}\left(G_{i-1}\right)$, which is a normal subgroup of $\operatorname{im}\left(\phi_{i}\right)$, and consists of a direct product of some of the direct factors of $\operatorname{im}\left(\phi_{i}\right)$, which are again easily identified. So we can assume that we have a representation of $Q_{i}$ as a direct product $S^{m}$ of a known number $m$ of copies of $S$.

Now, again by choosing random elements $g$ of $Q_{i}$, computing the orders of the components of $g$, and taking suitable powers of $g$, it is not difficult to find elements in the simple direct factors $S_{i}$ of $Q_{i}$, and hence to find generators of the $S_{i}$. We then carry out constructive recognition of the $S_{i}$ as discussed in Subsection 1.1. By checking that the relations of the presentation of the corresponding finite simple group are satisfied in $S_{i}$, we prove that $S_{i}$ is genuinely isomorphic to this simple group. So, if we had failed to find an Aschbacher decomposition for $S_{i}$ and wrongly concluded that $S_{i}$ was simple, then we would detect the error at this point. We would also find out at this stage if we had underestimated the number $m$ of copies of $S$, although that particular error is very unlikely indeed.

The constructive recognition of the $S_{i}$ enables us to solve the rewriting problem in $S_{i}$, and it is straightforward to glue these solutions together to solve the rewriting problem in $Q_{i}$. This process can be made more efficient by using the fact that the $S_{i}$ are permuted under the action of $G$ induced by conjugation in $G$ and the application of the map $\phi_{i}$. So, once we have solved the rewriting problem in one $S_{i}$, we can solve it immediately in any $S_{j}$ in the same orbit under this action of $G$. This refinement results in a very significant improvement in performance. We refer the reader to Section 2.3 of the second author's PhD thesis [31] for further details. 
Now we turn to the construction of a generating set of $\operatorname{ker}\left(\phi_{i}\right) \cap G_{i-1}$ modulo $O_{p}(G)$ in Step 3 of the process summarised earlier. We may construct individual random elements of $\operatorname{ker}\left(\phi_{i}\right) \cap G_{i-1}$ using the following method, which is described in $[23]$.

Let $g$ be a random element of $G_{i-1}$. Then we can compute $\phi_{i}(g) \in Q_{i}$. Let $\left\{x_{1}, \ldots, x_{k}\right\}$ be our generating set for $G_{i-1}$, so $Q_{i}$ is generated by $\left\{\phi_{i}\left(x_{1}\right), \ldots\right.$, $\left.\phi_{i}\left(x_{k}\right)\right\}$. If $Q_{i}$ is a soluble group defined by a polycyclic presentation, then we can use the standard methods for that class of groups to write $\phi_{i}(g)$ as a word $w\left(\phi_{i}\left(x_{1}\right), \ldots, \phi_{i}\left(x_{k}\right)\right)$; see, for example, [17, Section 8.3]. If $Q_{i}$ is insoluble, then we can use constructive recognition in $Q_{i}$ to do the same. Then $g w\left(x_{1}, \ldots, x_{k}\right)^{-1}$, which we shall call the residue of $g$ under $\phi_{i}$, is in the kernel of $\phi_{i}$ and it can be shown that, if $g$ is a uniformly distributed random element of $G_{i-1}$, then the residue of $g$ is a uniformly distributed random element of $\operatorname{ker}\left(\phi_{i}\right) \cap G_{i-1}$. By computing a sufficient number of residues of random elements of $G_{i-1}$, we hope to construct a generating set for $\operatorname{ker}\left(\phi_{i}\right) \cap G_{i-1}$ modulo $O_{p}(G)$.

We describe now how we estimate how many random elements of $G_{i-1}$ we need in order to have a high probability of generating $\operatorname{ker}\left(\phi_{i}\right) \cap G_{i-1}$ modulo $O_{p}(G)$ for $i<k$. For a finite group $G$, we define $P_{k}(G)$ to be the probability that $k$ uniformly randomly chosen elements of $G$ generate $G$. Then our problem reduces to finding a lower bound for $P_{k}\left(K_{i} / O_{p}(G)\right)$.

To do this, we make use of the superset $M=\left\{S_{1}^{e_{1}}, \ldots, S_{m}^{e_{m}}, \mathbb{Z}_{p_{1}}^{t_{1}}, \ldots, \mathbb{Z}_{p_{r}}^{t_{r}}\right\}$ of the composition factors of $G / O_{p}(G)$ that we were able to estimate after finding the preliminary sequence of maps, as described at the end of Subsection 2.1. After identifying the images $Q_{k}$ for $1 \leqslant k \leqslant i$, we can refine this to a superset of the composition factors of $K_{i} / O_{p}(G)$ by removing those factors that we know lie in one the $Q_{k}$ already found.

There are a number of expositions concerning this problem and most of the results referred to here are due to Pak (unpublished). We shall give only a brief outline here and refer to the reader to [31] for further details. Using elementary arguments, the following result can be proved, which reduces the problem to the case when $G$ is a direct product of isomorphic simple groups.

THEOREM 2.10. If $M$, as defined above, is a superset of the composition factors of $G$, then

$$
P_{k}(G) \geqslant \prod_{i=1}^{m} P_{k}\left(S_{i}^{e_{i}}\right) \cdot \prod_{i=1}^{r} P_{k}\left(\mathbb{Z}_{p_{i}}^{t_{i}}\right) .
$$

For the abelian case, it can be shown that

$$
P_{k}\left(\mathbb{Z}_{p}^{t}\right)=\prod_{j=1}^{t}\left(1-\frac{p^{j-1}}{p^{k}}\right)
$$

and for the nonabelian case we have

$$
P_{k}\left(S^{e}\right)=P_{k}^{e}(S) \prod_{j=1}^{e-1}\left(1-\frac{j|\operatorname{Out}(S)|}{P_{k}(S)|S|^{k-1}}\right) .
$$

Because we have identified the nonabelian composition factors of $G$ nonconstructively, we can assume that each $|\operatorname{Out}(S)|$ is known, and it is easy to see that

$$
P_{k}(S) \geqslant 1-\left(1-P_{2}(S)\right)^{\left[\frac{k}{2}\right]},
$$


so it remains only to estimate $P_{2}(S)$. For this, we are forced to rely on the following conjecture

CONJECTURE 2.11. Let $S$ be a nonabelian finite simple group. Then

$$
P_{2}(S) \geqslant P_{2}(\operatorname{Alt}(6))=\frac{53}{90} .
$$

This has not yet been proved, but it is known that $P_{2}(S) \rightarrow 1$ as $|S| \rightarrow \infty$ (see $[\mathbf{2 6}]$ ), and an examination of the simple groups up to order $10^{6}$ indicates that the probability is much closer to 1 than $\frac{53}{90}$ for all but the smallest of the simple groups, so we are highly confident that this conjecture is true.

So, putting all of this together, we have

THEOREM 2.12. Given a superset of the multiset of composition factors of a group $G$ and $\epsilon>0$ we can find $k$ such that $P_{k}(G) \geqslant 1-\epsilon$.

This enables us to compute a reasonable estimate for the number of random elements that are required to generate the kernel of any of the $\phi_{i}$ modulo $O_{p}(G)$ for $i<k$.

\subsection{Verifying the kernels}

In Subsection 2.3 we constructed a chain of subgroups $G=G_{0} \geqslant G_{1} \geqslant \cdots \geqslant$ $G_{k-1}$ with $G_{i-1} \leqslant \operatorname{Domain}\left(\phi_{i}\right)$ and we identified the images $Q_{i}=\phi_{i}\left(G_{i-1}\right)$ for $1 \leqslant i \leqslant k$. From the construction we know that $G_{i} \leqslant \operatorname{ker}\left(\phi_{i}\right)$, and we want to verify that $G_{i} O_{p}(G)=\left(\operatorname{ker}\left(\phi_{i}\right) \cap G_{i-1}\right) O_{p}(G)$ for $1 \leqslant i<k$. We shall also explain in this subsection how to test elements of $\operatorname{GL}(n, q)$ for membership of the subgroups $G_{i}$.

We make use of the following theorem.

THEOREM 2.13. Let $G=\langle\Gamma\rangle$, and let $\pi: G \rightarrow Q$ be an epimorphism. Let $\langle\bar{X} \mid \bar{R}\rangle$ be a presentation for $Q$ and, for each $h \in Q$, let $w_{\bar{X}}(h)$ be a word for $h$ over $\bar{X}$.

For each $\bar{x} \in \bar{X}$ choose $x \in G$ with $\pi(x)=\bar{x}$. Define a map $\theta: F_{\bar{X}} \rightarrow G$ (where $F_{\bar{X}}$ is the free group on $\left.\bar{X}\right)$ by extending the map $\bar{x} \mapsto x$.

Let $K=\langle Y\rangle$ be a subgroup of $\operatorname{ker}(\pi)$. Then $K=\operatorname{ker}(\pi)$ if and only if the following hold:

(i) $g \cdot \theta\left(w_{\bar{X}}(\pi(g))\right)^{-1} \in K$ for all $g \in \Gamma$;

(ii) $\theta(\bar{r}) \in K$ for all $\bar{r} \in \bar{R}$;

(iii) $y^{g} \in K$ for all $g \in \Gamma$ and $y \in Y$.

Proof. Suppose that the conditions (i), (ii) and (iii) are satisfied. Then (iii) says that $K \unlhd G$, and (i) says that $g \equiv \theta\left(w_{\bar{X}}(\pi(g))\right) \bmod K$ for all $g \in \Gamma$. So, for $g=g_{1}^{\epsilon_{1}} \cdots g_{r}^{\epsilon_{r}} \in G$ with $g_{i} \in \Gamma$ and $\epsilon_{i}= \pm 1$, we have

$$
g \equiv \theta\left(w_{\bar{X}}\left(\pi\left(g_{1}\right)\right)^{\epsilon_{1}} \cdots w_{\bar{X}}\left(\pi\left(g_{r}\right)\right)^{\epsilon_{r}}\right) \bmod K .
$$

If $g \in \operatorname{ker}(\pi)$, then (since $K \leqslant \operatorname{ker}(\pi)), w_{\bar{X}}\left(\pi\left(g_{1}\right)\right)^{\epsilon_{1}} \cdots w_{\bar{X}}\left(\pi\left(g_{r}\right)\right)^{\epsilon_{r}}$ is a product in $F_{\bar{X}}$ of conjugates of elements of $\bar{R}$ and their inverses. But, by (ii), $\theta(\bar{r}) \equiv 1 \bmod$ $K$ for each $\bar{r} \in \bar{R}$, so $\theta\left(w_{\bar{X}}\left(\pi\left(g_{1}\right)\right)^{\epsilon_{1}} \cdots w_{\bar{X}}\left(\pi\left(g_{r}\right)\right)^{\epsilon_{r}}\right) \equiv 1 \bmod K$ and hence $g \in K$ and $K=\operatorname{ker}(\pi)$.

Conversely, it is easy to see that if $K=\operatorname{ker}(\pi)$ then the three conditions hold. 
We shall apply this result with $\pi=\left.\phi_{i}\right|_{G_{i-1}}, G=G_{i-1}, Q=Q_{i}$ and $K=$ $G_{i}$, but we shall check that the elements to be tested in (i), (ii) and (iii) lie in $G_{i} O_{p}(G)$, thereby proving that $G_{i} O_{p}(G)=\left(\operatorname{ker}\left(\phi_{i}\right) \cap G_{i-1}\right) O_{p}(G)$. (So we are actually applying the theorem in $G_{i-1} O_{p}(G) / O_{p}(G)$.)

For this purpose, we need to be able to test elements of $\operatorname{GL}(n, q)$ for membership in the subgroups $G_{i} O_{p}(G)$. We shall describe now how we can use the information computed so far to carry out this membership testing, but we observe that this method requires us to complete the computation of all of the images and kernels before we attempt the verification process.

We can perform membership testing in $O_{p}(G)$ as follows. If $G$ is irreducible then $O_{p}(G)=1$, so assume $G$ to be reducible with $m$ irreducible blocks of dimensions $d_{1}, \ldots, d_{m}$. Using the Meataxe we compute a matrix $x$ (in fact such a matrix has already been computed during the application of REDUCIBLEMAPS to $G$ ) such that elements $g \in G$ conjugated by $x$ have the form

$$
\left(\begin{array}{ccc}
g_{1} & & \\
& \ddots & 0 \\
* & & g_{m}
\end{array}\right),
$$

for $g_{i} \in \mathrm{GL}\left(d_{i}, q\right)$. Then $g \in O_{p}(G)$ if and only if $g_{i}=I_{d_{i}}$ for $1 \leqslant i \leqslant m$.

We now test membership in $G_{k-1} O_{p}(G)$ as follows. An element $g \in \operatorname{Domain}\left(\phi_{k}\right)$ belongs to $G_{k-1} O_{p}(G)$ if and only if $\phi_{k}(g) \in Q_{k}$ and the residue of $g$ (as defined in Subsection 2.3) under $\phi_{k}$ belongs to $O_{p}(G)$. (This assumes that we can test elements of $\operatorname{GL}(n, q)$ for membership of the domains of the maps $\phi_{i}$, but this presents no difficulty. Most of the maps are based on Aschbacher decompositions, and testing for membership of $g$ in Domain $\left(\phi_{i}\right)$ is equivalent to checking that $g$ preserves the decomposition in question.)

Using Theorem 2.13 we can now verify $G_{k-1} O_{p}(G)=\left(\operatorname{ker}\left(\phi_{k-1}\right) \cap G_{k-2}\right) O_{p}(G)$. This, in turn, enables us to test membership in $G_{k-2} O_{p}(G)$ : an element $g \in$ $\operatorname{Domain}\left(\phi_{k-1}\right)$ belongs to $G_{k-2} O_{p}(G)$ if and only if $\phi_{k-1}(g) \in Q_{k}$ and the residue of $g$ under $\phi_{k-1}$ belongs to $G_{k-1} O_{p}(G)$.

We repeat this process to produce membership tests for $G_{i} O_{p}(G)$ for $i=k-1$, $k-2, \ldots, 1,0$, and to verify that $G_{i} O_{p}(G)=\left(\operatorname{ker}\left(\phi_{i}\right) \cap G_{i-1}\right) O_{p}(G)$ for $i>0$. If at any stage this process fails and we find elements in $\left(\operatorname{ker}\left(\phi_{i}\right) \cap G_{i-1}\right) \backslash G_{i} O_{p}(G)$, then we add these elements to the generating set for $G_{i}$ and recompute the series from this point.

It is possible, at the same time, to use the presentations of the $Q_{i}$ and the conjugation action of $G_{i}$ on $G_{i-1}$ to compute presentations of $G_{i} O_{p}(G) / O_{p}(G)$ for $i=k-1, k-2, \ldots, 0$ (see [17, Subsection 2.4.3] for the theory of this). We need to do this if we wish to prove the correctness of the structure af $O_{p}(G)$, which we shall be discussing in the next subsection.

\subsection{From $O_{p}(G)$ to 1}

We now have a normal series for $G \leqslant \operatorname{GL}(n, q)$, with $q=p^{e}$, with last term $O_{p}(G)$. To complete our analysis of $G$, we need to construct a chain of subgroups

$$
1 \leqslant H_{r} \leqslant H_{r-1} \leqslant \cdots \leqslant H_{0}=O_{p}(G)
$$

with each $H_{i} \unlhd G$ and each $H_{i} / H_{i+1}$ an elementary abelian $p$-group. This can be difficult in matrix groups of dimension greater than about 100, and this topic 
remains a challenging area of research. Here we shall give only a summary of the methods that we have used to date.

If $G$ is irreducible then $O_{p}(G)=1$, so assume that $G$ is reducible with $m$ blocks of dimensions $d_{1}, \ldots, d_{m}$. We are already in possession of a basis change matrix $x$ such that elements of $G^{x}$ have the form

$$
\left(\begin{array}{ccccc}
g_{1} & & & & \\
b_{2,1} & g_{2} & & 0 & \\
b_{3,1} & b_{3,2} & \ddots & & \\
\vdots & \vdots & \ddots & g_{m-1} & \\
b_{m, 1} & b_{m, 2} & \cdots & b_{m, m-1} & g_{m}
\end{array}\right),
$$

where $g_{i}$ is a $d_{i} \times d_{i}$ matrix and $b_{i, j}$ is a $d_{i} \times d_{j}$ matrix.

Then $O_{p}(G)$ consists of those elements of $G$ for which $g_{i}=I_{d_{i}}$ for $1 \leqslant i \leqslant m$, and we let $H_{0}=O_{p}(G)$. We define $H_{1}$ to be the subgroup of $H_{0}$ consisting of those matrices in which the blocks $b_{2,1}, b_{3,2}, \ldots, b_{m, m-1}$ immediately below the main diagonal are all 0 . Then the reader can verify that $H_{1} \unlhd G$ and $H_{0} / H_{1}$ is an elementary abelian $p$-group. Furthermore, we can regard $H_{0} / H_{1}$ as a module for $G / O_{p}(G)$ over $\mathbb{F}_{p}$, and it is a submodule of the module of dimension $e\left(d_{1} d_{2}+d_{2} d_{3}+\right.$ $\left.\cdots+d_{m-1} d_{m}\right)$ which is defined by all matrices having this form.

Similarly, for $1 \leqslant k \leqslant m-2$, we define $H_{k}$ to be the subgroup of $H_{0}$ consisting of those matrices in which $b_{i, j}=0$ whenever $i-j \leqslant k$ and put $H_{m-1}=1$. Then each $H_{i} \unlhd G$, and $H_{i-1} / H_{i}$ is an elementary abelian $p$-group and can be regarded as a module for $G / O_{p}(G)$ over $\mathbb{F}_{p}$.

The difficulty in analysing $O_{p}(G)$ arises from the fact that a minimal generating set for $O_{p}(G)$ can be inconveniently large. For example, $O_{p}(G)$ requires $n^{2} / 4$ generators in a maximal reducible subgroup of $\operatorname{GL}(n, p)$ in which $d_{1}=d_{2}=n / 2$. This can make it impractical to store a full generating set of matrices for $O_{p}(G)$ when $n$ is larger than about 100, since the storage requirements become excessive.

Instead, we store only enough matrices in each $H_{i-1}$, for $1 \leqslant i<m$, to generate $H_{i-1} / H_{i}$ as a $G / O_{p}(G)$-module. (In other words, we store elements that generate $H_{i-1}$ modulo $H_{i}$ as a normal subgroup of $G$.) We can then use the SPINBASIS algorithm described in [17, Section 7.4] to find the dimension of $H_{i-1} / H_{i}$ as a module, and hence find the order of $\left|H_{i-1} / H_{i}\right|$. SPINBASIs is practical for modules of dimension several thousand and has been used successfully for dimensions up to 100000 , so this approach can significantly increase the range of practicality of the methods.

To start off these calculations, we find random elements of $H_{0}=O_{p}(G)$ by calculating the residues of random elements of $G$ under $\phi_{i}$ for $i=1,2, \ldots, k$ in turn. After finding what we hope are enough such elements to generate $H_{0} / H_{1}$ as a $G / O_{p}(G)$-module, we apply SpINBAsis to these elements. To verify that we have now successfully generated the complete submodule $H_{0} / H_{1}$, it is most convenient to use a presentation of $G / O_{p}(G)$, the calculation of which was described at the end of the last subsection. We use Theorem 2.13 and check that all relators of $G / O_{p}(G)$ and the residues of all generators of $G$ under $\phi_{i}$ for $i=1,2, \ldots, k$ lie in the submodule $H_{0} / H_{1}$ that we have calculated. If not, then we introduce the offending elements as new module generators of $H_{0} / H_{1}$ and re-apply SPINBASIS.

This method is particularly effective when $m=2$ and so $H_{1}=1$ and $O_{p}(G)$ is already elementary abelian, since then the calculation above completes the anal- 
ysis of $O_{p}(G)$, and there is no need to compute a complete generating set for $H_{0}$ as elements of $G$. When $m>2$, we need such a generating set for $H_{i-1}$ in order to find random elements of $H_{i}$, and in order to compute residues during the verification process. However, the generators of $H_{i-1}$ that are calculated as vectors during the application of SPINBASIS can be stored as words, which are products of conjugates by generators of $G$ of the module generators, rather than as matrices. It is straightforward to adapt SPINBASIs to compute these words.

There are many possible improvements to the basic outline of the approach that we have described here, and some of these are being investigated using experimental implementations. In many examples, the $H_{i-1} / H_{i}$ can be regarded as modules over $\mathbb{F}_{q}$ rather than just over $\mathbb{F}_{p}$, which renders module computations much more efficient when $q=p^{e}$ for $e>1$. We should also make use of the fact that the full modules, of which $H_{i-1} / H_{i}$ are submodules, are direct sums arising in the obvious way from the individual blocks $B_{i, j}$.

\subsection{Refining the Normal Series}

We have now computed a normal series for $G$ in which the factors are either soluble groups or direct products of isomorphic non-abelian simple groups. Refining this series to a chief series of $G$ is straightforward, and we shall describe how to do this only briefly.

A non-abelian layer in the series is of the form $K_{i-1} / K_{i} \cong S^{m}$, where the $m$ direct factors in the product $S^{m}$ are permuted under the conjugation action of $G$. The new subgroups that we need to introduce to refine the series correspond to the orbits of this action on these $m$ factors, which are easily computed using the map $\phi_{i}$.

The soluble layers are either the elementary abelian $p$-groups $H_{i-1} / H_{i}$ within $O_{p}(G)$ that were discussed in the previous subsection, or are defined by means of a polycyclic presentation. In the second of these cases we can compute a series of characteristic subgroups with elementary abelian layers, thereby effectively reducing the problem to the case when the layer is an elementary abelian $r$-group for some prime $r$. We can then use the conjugation action of $G$ to make this layer into a module for $G$ over $\mathbb{F}_{r}$, and use the Meataxe [17, Section 7.4] to find a composition series for the module. The terms in this series correspond to the required refinement of the layer in $G$.

\section{Rearranging the chief series}

At this stage, we have computed a chief series

$$
G=G_{0} \geqslant G_{1} \geqslant \cdots \geqslant G_{n}=1
$$

of our given group $G \leqslant \operatorname{GL}(n, q)$, and our final objective is to replace it with a new chief series that passes through the subgroups $O_{\infty}(G), \operatorname{soc}^{*}(G)$ and $\operatorname{Pker}(G)$ that were defined in Section 1. The proof of the following lemma is straightforward, given that these are characteristic subgroups of $G$.

LEMMA 3.1. If $N$ is any normal subgroup of any finite group $G$, then $O_{\infty}(N)=$ $O_{\infty}(G) \cap N, \operatorname{soc}^{*}(N)=\operatorname{soc}^{*}(G) \cap N$ and $\operatorname{Pker}(N)=\operatorname{Pker}(G) \cap N$,

The method described for rearranging the series in [31], which corresponds to the second author's implementation, involves considering each pair of adjacent chief 
factors in the series and interchanging factors firstly in order to bring the soluble factors as low down in the series as possible, which results in the series passing through $O_{\infty}(G)$, and then secondly to bring the insoluble factors as low down as possible in the series modulo $O_{\infty}(G)$, which results in the series passing through $\operatorname{soc}^{*}(G)$, and finally bringing the factors lying in $\operatorname{Pker}(N)$ down to the bottom of the series modulo $\operatorname{soc}^{*}(G)$. Here we shall describe a variation of this procedure which achieves all three objectives in a single pass upwards through the chief factors, and which we believe will turn out to be more efficient.

\subsection{Identifying inner automorphisms}

The rearranging process depends critically on our ability to solve the following algorithmic problem efficiently.

Problem 3.2. Given a finite nonabelian simple group $S$ and an automorphism $\sigma$ of $S$, determine whether $\sigma$ is an inner automorphism and, if so, find $g \in S$ such that $\sigma$ is conjugation by $g$.

We assume that $g^{\sigma}$ is easily computable for $g \in S$. In our application, $\sigma$ will be induced by conjugation by an element of a larger group. We assume also that we have solved the constructive recognition problem for $S$, which enables us to work within the standard copy $\hat{S}$ of $S$.

If $\hat{X}$ is a generating set for $\hat{S}$, then the problem is equivalent to the following. Does there exist $g \in \hat{S}$ with $x^{g}=x^{\sigma}$ for all $x \in \hat{X}$ ? If $\hat{S}$ is a permutation group, then we can solve this by a sequence of conjugacy tests and centraliser calculations within $\hat{S}$ and subgroups of $\hat{S}$.

So we shall assume that $\hat{S}$ is a quasisimple absolutely irreducible subgroup of $\operatorname{GL}(d, r)$ for some $d$ and $r$. We then have the additional complication that $\hat{S}$ may contains scalars with $\hat{S} / Z(\hat{S}) \cong S$. So, given a generating set $\hat{X}$ of $\hat{S}$, we are looking for an element $g \in \hat{S}$ with $x^{g} \equiv x^{\sigma} \bmod Z(\hat{S})$ for all $x \in \hat{X}$. However, it is readily checked that every finite simple group $S$ is divisible by a prime which does not divide the order of the Schur multiplier of $S$, so we can choose $\hat{X}$ to consist of elements with order coprime to $|Z(\hat{S})|$ and then the problem reverts to finding $g \in \hat{S}$ with $x^{g}=x^{\sigma}$ for all $x \in \hat{X}$.

Deciding whether there exists $g \in \mathrm{GL}(d, r)$ with $x^{g}=x^{\sigma}$ for all $x \in \hat{X}$ is equivalent to testing the $\hat{S}$-modules defined by the matrices in $\hat{X}$ and in $\hat{X}^{\sigma}$ for isomorphism, and this can be done readily using the algorithm described in $[\mathbf{1 7}$, Section 7.5.3]. Since $\hat{S}$ is absolutely irreducible, if $g$ exists then it is unique modulo scalars, and so we can complete the test by checking whether $g z$ lies in $\hat{S}$ for some scalar matrix $z$, which we are able to do as part of the defining assumptions of the standard copy of a simple group.

\section{2. $\quad$ Moving chief factors}

Now we present a brief summary of how we rearrange the chief series of $G$ to pass through the three characteristic subgroup defined above.

Definition 3.3. We say that a chief factor $G_{i-1} / G_{i}$ of $G$ belongs to (or lies in) a normal subgroup $N$ of $G$ if $G_{i-1} \leqslant N G_{i}$.

Our aim is to rearrange the chief factors of $G$ such that those that belong to $O_{\infty}(G)$ occur first, followed by all others that belong to $\operatorname{soc}^{*}(G)$, then by all others that belong to $\operatorname{Pker}(G)$, and finally by those not belonging to $\operatorname{Pker}(G)$. 
We consider the chief factors $G_{i-1} / G_{i}$ for $i=n, n-1, \ldots, 1$ in turn. If this factor is soluble then we decide whether we can rearrange the series so that it belongs to $O_{\infty}(G)$ and, if so, then we carry out this rearrangement within $G_{i-1}$. If not, then we consider it for membership of $\operatorname{Pker}(G)$ and, if this is the case, then we move it down into $\operatorname{Pker}\left(G_{i-1}\right)$. If $G_{i-1} / G_{i}$ is insoluble, then we decide whether we can rearrange the series so that this chief factor belongs to $\operatorname{soc}^{*}(G)$ and, if so, rearrange the series accordingly.

So, when we come to consider $G_{i-1} / G_{i}$, we can assume that those chief factors of $G$ that lie in $G_{i}$ have already been rearranged to occur in the required order. In other words, the series for $G_{i}$ passes through $O_{\infty}\left(G_{i}\right)=O_{\infty}(G) \cap G_{i}, \operatorname{soc}^{*}\left(G_{i}\right)=$ $\operatorname{soc}^{*}(G) \cap G_{i}$ and $\operatorname{Pker}\left(G_{i}\right)=\operatorname{Pker}(G) \cap G_{i}$. We assume, in addition, that we have stored the following extra information during the analysis of the chief factors of $G$ within $G_{i}$. For those factors in $\operatorname{Pker}\left(G_{i}\right)$ but not in $\operatorname{soc}^{*}\left(G_{i}\right)$, we store the outer automorphisms of the simple factors of $\operatorname{soc}^{*}\left(G_{i}\right) / O_{\infty}\left(G_{i}\right)$ that are induced by the generators of these chief factors. For those that lie outside of $\operatorname{Pker}\left(G_{i}\right)$, we store the permutations of the simple factors of $\operatorname{soc}^{*}\left(G_{i}\right) / O_{\infty}\left(G_{i}\right)$ that are induced by the conjugation action of their generators.

(Computing within the permutation group $G_{i} / \operatorname{Pker}\left(G_{i}\right)$ is easy, but we are assuming also that we can compute effectively within the outer automorphism groups of the finite nonabelian simple groups. Since these are all relatively small soluble groups, this is a reasonable assumption. Indeed, we have already implemented this part of the proces for the classical groups.)

We now explain how we analyse the next chief factor $G_{i-1} / G_{i}$. We already know a set of elements $X_{i-1}$ of $G_{i-1}$ that generate $G_{i-1}$ modulo $G_{i}$. During the analysis, we may change this set $X_{i-1}$ by multiplying its members by suitable elements of $G_{i-1}$ for the purpose of making $X_{i-1}$ a subset of $O_{\infty}\left(G_{i-1}\right)$, $\operatorname{soc}^{*}\left(G_{i-1}\right)$, or $\operatorname{Pker}\left(G_{i-1}\right)$ whenever this is possible.

Step 1: does the factor lie in $\operatorname{Pker}(G)$ ? Let $x$ be the first element of $X_{i-1}$. We first test whether the conjugation action of $x$ on the simple factors of $\operatorname{soc}^{*}\left(G_{i}\right) / O_{\infty}\left(G_{i}\right)$ lies within the permutation group on these factors that we have stored already for $G_{i}$. If not, then $G_{i-1} / G_{i}$ does not lie in $\operatorname{Pker}(G)$, and we compute and store the permutations of these simple factors induced by the elements of $X_{i-1}$. If it is then, since $G_{i-1} / G_{i}$ is a chief factor of $G$, the same will be true for each element of $X_{i-1}$, and we multiply each such element by the inverse of an element of $G_{i}$ that induces the same permutation and thereby effectively move $G_{i-1} / G_{i}$ into $\operatorname{Pker}\left(G_{i-1}\right)$.

Step 2: does it lie in $\operatorname{soc}^{*}(G)$ ? If the chief factor lies in $\operatorname{Pker}\left(G_{i-1}\right)$, then we test whether the outer automorphisms of the simple factors of $\operatorname{soc}^{*}\left(G_{i}\right) / O_{\infty}\left(G_{i}\right)$ all lie in the subgroups of these outer automorphism groups that we have stored already for $G_{i}$. If not, then $G_{i-1} / G_{i}$ is not a chief factor of $\operatorname{soc}^{*}(G)$, and we compute and store the outer automorphisms of the simple factors induced by the elements of $X_{i-1}$. If it is (which will necessarily be the case if $G_{i-1} / G_{i}$ is not soluble), then the same will be true for each element of $X_{i-1}$, and we replace each such element by the result of multiplying it by the inverse of an element of $G_{i}$ that induces the same outer automorphism. We thereby move $G_{i-1} / G_{i}$ into $\operatorname{soc}^{*}\left(G_{i-1}\right)$.

Step 3: does it lie in $O_{\infty}(G)$ ? If the chief factor now lies in $\operatorname{soc}^{*}\left(G_{i-1}\right)$, then the elements of $X_{i-1}$ all induce inner automorphisms of the simple factors of $\operatorname{soc}^{*}\left(G_{i}\right) / O_{\infty}\left(G_{i}\right)$, and we can identify the corresponding conjugating elements of these simple factors using the method described in Subsection 3.1. We then replace each element of $X_{i-1}$ by the result of multiplying it by the inverse of an appropriate 
element of $\operatorname{soc}^{*}\left(G_{i}\right)$, after which the elements of $X_{i-1}$ will all centralise $\operatorname{soc}^{*}\left(G_{i}\right)$ modulo $O_{\infty}\left(G_{i}\right)$. So now, if $G_{i-1} / G_{i}$ is soluble, then we will have effectively moved it into $O_{\infty}\left(G_{i-1}\right)$, whereas if it is insoluble, we will have achieved the desirable effect of making the group generated by $X_{i-1}$ modulo $O_{\infty}\left(G_{i-1}\right)=O_{\infty}\left(G_{i}\right)$ equal to some of the simple factors of $\operatorname{soc}^{*}\left(G_{i}\right) / O_{\infty}\left(G_{i}\right)$.

\section{Some timings}

To conclude, we present some timings for the second author's MAGMA implementation of the algorithms described in Sections 2 and 3. The computations were run on an AMD Opteron Model 152 processor running at 2.6 GH with 4 GB of memory. The times in the columns labelled CSTime and SSTime in the table are respectively for computing a chief series, and a chief series passing through the three subgroups $O_{\infty}(G)$, $\operatorname{soc}^{*}(G)$ and $\operatorname{Pker}(G)$. However, the latter times also include some additional computations, such as polycyclic presentations of $O_{\infty}(G)$, which accounts for the longer time in the soluble example $\left(\mathrm{GL}(2,3)<S_{4}\right)$ ₹ $S_{4}$.

It should be noted that presentations for the larger sporadic simple groups are currently unavailable in MAGMA, so the complete verification algorithms of Section 2.4 have not been run on these groups. Unfortunately, implementations of constructive recognition algorithms were only available for $\operatorname{PSL}(2, q), \operatorname{Sz}(q)$ and $\operatorname{Alt}(n)$ and most of the sporadic groups, and we had to use the default Schreier-Sims algorithm for other finite simple groups. This considerably restricts the scope of the current implementation, and we hope to extend this scope eventually by making making use of further constructive recognition methods. In particular, the simple groups $\operatorname{PSL}\left(3,3^{7}\right)$ and $\mathrm{P} \Omega(9,9)$, which occur as composition factors of examples in the table, are about at the limit of what we can handle at present.

\begin{tabular}{|c|c|c|c|c|}
\hline $\mathrm{G}$ & d & $\mathbf{q}$ & CSTime & SSTime \\
\hline $3^{1+12} \cdot 2 \mathrm{Suz} .2$ & 78 & 3 & 4.5 & 4.5 \\
\hline $2^{576} \cdot \mathrm{Co}_{1} 2 S_{2}$ & 96 & 2 & 13.4 & 48.5 \\
\hline GL $(6,5)$ \ $\operatorname{Sym}(15)$ & 90 & 5 & 14.5 & 31.3 \\
\hline$\left(\mathrm{GL}(2,3) \backslash S_{4}\right) \backslash S_{4}$ & 32 & 3 & 1.8 & 40.7 \\
\hline $\operatorname{Ly} r S_{2}$ & 222 & 5 & 33.9 & 38.4 \\
\hline $3^{18} . \operatorname{PSL}(3,7)^{6} .9 .2$ & 18 & 7 & 4.3 & 5.2 \\
\hline $2^{32} \cdot \mathrm{SL}(4,5) \cdot 2^{4} \cdot \mathrm{GL}(2,4)$ & 64 & 5 & 4.3 & 4.4 \\
\hline $\operatorname{GL}\left(3,3^{7}\right) .7$ & 21 & 3 & 89.9 & 89.9 \\
\hline $\mathrm{SO}(9,9) .2$ & 18 & 3 & 157.5 & 158.0 \\
\hline $2^{1+14} \cdot \operatorname{Sp}(14,2)$ & 128 & 5 & 18.2 & 18.5 \\
\hline $\mathrm{GL}(3,7) \otimes 2 S_{5}$ & 243 & 7 & 100.4 & 121.0 \\
\hline $3^{1+6} \cdot \operatorname{Sp}(6,3) \otimes \operatorname{GL}(5,5)$ & 135 & 25 & 97.5 & 109.5 \\
\hline$(\mathrm{GL}(2,5) \otimes \mathrm{GL}(3,5)) \backslash S_{15}$ & 90 & 5 & 19.2 & 97.2 \\
\hline
\end{tabular}

Table 1: Table of timings for computing SR-Data

Here is a brief description of the examples in the table. The first two are reducible groups in which $O_{p}(G)$ is non-trivial. The second of these also involves the imprimitive matrix group $\mathrm{Co}_{1} 2 S_{2}$ of degree 48. In both of these two examples, REDUCIBLEMAPS duplicated effort by analysing the same nonabelian chief factor on different irreducible constituents of the input group. 
The following five examples are all imprimitive, with $3^{18}$. PSL $(3,7)^{6} .9 .2 \leqslant$ $\left(\operatorname{SL}(3,7) \gtrless S_{2}\right) \gtrless D_{18}$ and $2^{32} . \operatorname{PSL}(4,5) \cdot 2^{4} . \operatorname{GL}(2,4) \leqslant \operatorname{SL}(4,5) \gtrless \operatorname{AGL}(2,4)$. The next two examples $\mathrm{GL}\left(3,3^{7}\right) .7$ and $\mathrm{SO}(9,9) .2$ are semilinear, $2^{1+14} \cdot \mathrm{Sp}(14,2)$ is the normaliser of a symplectic type 2 -group and $\mathrm{GL}(3,7) \otimes 2 S_{5}$ is tensor induced. The example $3^{1+6} \cdot \operatorname{Sp}(6,3) \otimes \mathrm{GL}(5,5)$ is a tensor product of a normaliser of an extraspecial group with $\mathrm{GL}(5,5)$, and $(\mathrm{GL}(2,5) \otimes \mathrm{GL}(3,5))$ ? $S_{15}$ is imprimitive with kernel 15 copies of the tensor product $\mathrm{GL}(2,5) \otimes \mathrm{GL}(3,5)$.

\section{References}

1. M. AschBACHER, 'On the maximal subgroups of the finite classical groups', Invent. Math. 76 (1984) 469-514. 226

2. H. B ̈̈̈̈RNHIELM, 'Recognising the Ree groups in their natural representations', Preprint. 226

3. H. B ̈̈̈̈RNHIELM, 'Recognising the Suzuki groups in their natural representations', J. Algebra 300 (2006) 171-198. 226

4. L. Babai and A. Shalev, 'Recognizing simplicity of black-box groups and the frequency of $p$-singular elements in affine groups', Groups and Computation III, Ohio State Univ. Math. Res. Inst. Publ. 8 (de Gruyter, Berlin, 2001) 39-62. 238

5. LÁsZLÓ BABAI, 'Local expansion of vertex-transitive graphs and random generation in finite groups', Theory of Computing, Los Angeles, 1991 (Association for Computing Machinery, New York, 1991) pp. 164-174. 224

6. S. BRATUs and I. PAK, 'Fast constructive recognition of a black box group isomorphic to $A_{n}$ or $S_{n}$ using Goldbach's conjecture', J. Symbolic Comput. 29 (2000) 33-57. 226

7. J.J. Cannon, B.C. Cox and D.F. Holt, 'Computing chief series, composition series and socles in large permutation groups', J. Symbolic Comput. 24 (1997) 285-301. 229

8. J.J. CAnnon and D.F. Holt, 'Automorphism group computation and isomorphism testing in finite groups', J. Symbolic Comput. 35 (2003) 241-267. 223

9. J.J. CAnnon and D.F. Holt, 'Computing maximal subgroups of finite groups', J. Symbolic Comput. 37 (2004) 598-609. 223

10. J.J. CAnnon and D.F. Holt, 'Computing conjugacy class representatives in permutation groups', J. Algebra 300 (2006) 213-222. 223

11. F. Celler, C.R. Leedham-Green, S.H. Murray, A.C. Niemeyer and E.A. O'Brien, 'Generating random elements of a finite group', Comm. Algebra 23 (1995) 4931-4948. 225

12. Frank Celler and C.R. Leedham-Green, 'Calculating the order of an invertible matrix', Groups and Computation II (DIMACS, 1995), Amer. Math. Soc. DIMACS Series 28 (American Mathematical Society, Providence, RI, 1997) 55-60. 224

13. M.D.E. Conder, C.R. Leedham-Green and E.A. O'Brien, 'Constructive recognition of PSL(2,q)', Trans. Amer. Math. Soc. 358 (2006) 1203-1221. 226 
14. S.P. Glasby, C.R. Leedham-Green and E.A. O'Brien, 'Writing projective representations over subfields', J. Algebra 295 (2006) 1203-1221. 233

15. D. Gorenstein, Finite Groups (Harper \& Row, New York, Evanston, London, 1968). 233, 234

16. P.E. Holmes, S.A. Linton, E.A. O'Brien, A.J.E. Ryba and R.A. Wilson, 'Constructive membership testing in black-box groups', Preprint. 226

17. Derek F. Holt, Bettina Eick and Eamonn A. O'Brien, Handbook of computational group theory (Chapman and Hall/CRC, London, 2005). 223, $224,225,226,241,243,244,245,246$

18. Derek F. Holt, C.R. Leedham-Green, E.A. O'Brien and Sarah Rees, 'Computing matrix group decompositions with respect to a normal subgroup', J. Algebra 184 (1996) 818-838. 234, 235

19. Derek F. Holt, C.R. Leedham-Green, E.A. O'Brien and Sarah Rees, 'Testing matrix groups for primitivity', J. Algebra 184 (1996) 795-817. 231

20. Derek F. Holt and SARAh ReEs, 'Testing modules for irreducibility', J. Austral. Math. Soc. Ser. A 57 (1994) 1-16. 232

21. W.M. Kantor and À. Seress, 'Black box classical groups', Mem. Amer. Math. Soc. 149 (2001), no. 708. 226

22. P. Kleidman and M. Liebeck, The Subgroup Structure of the Finite Classical Groups, London Mathematical Society Lecture Notes Series 129 (Cambridge University Press, Cambridge, 1990). 236

23. C.R. Leedham-Green, 'The computational matrix group project', Groups and Computation III, Columbus, OH, 1999 (de Gruyter, Berlin, 2001) 229248. 223, 231, 241

24. C.R. Leedham-Green and E.A. O'Brien, 'Recognising tensor products of matrix groups', Internat. J. Algebra Comput. 7 (1997) 541-559. 233

25. C.R. Leedham-Green and E.A. O'Brien, 'Recognising tensor induced matrix groups', J. Algebra 253 (2002) 14-30. 236, 238

26. M. Liebeck and A. Shalev, 'The probability of generating a finite simple group', Geom. Dedicata 56 (1995) 103-113. 242

27. F. Luebeck, K. MagaArd and E.A. O'Brien, 'Constructive recognition of $\mathrm{SL}_{3}(q)^{\prime}$, Preprint. 226

28. Alice C. Niemeyer and Cheryl E. Praeger, 'A recognition algorithm for classical groups over finite fields', Proc. London Math. Soc. (3) 77 (1998) 117-169. 236

29. E.A. O'Brien, 'Towards effective algorithms for linear groups', Finite Geometries, Groups and Computation, Colorado, 2004 (de Gruyter, Berlin, 2006) 163-190. 223, 225

30. À. Seress, Permutation Group Algorithms (Cambridge University Press, Cambridge, 2003). 225

31. Mark Stather, 'Algorithms for computing with finite matrix groups', $\mathrm{PhD}$ thesis, University of Warwick, 2006. 224, 240, 241, 245 
Chief series and the soluble radical of a matrix group over a finite field

32. M.J. Stather, 'Sylow subgroups in matrix groups', J. Algebra, to appear. 224

33. D.E. TAYlor, The Geometry of the Classical Groups, Sigma Series in Pure Mathematics 9 (Heldermann, Berlin, 1992). 236

Derek F. Holt dfh@maths.warwick.ac.uk

Mark J. Stather markstather@hotmail.com

Mathematics Institute

University of Warwick

Coventry

CV4 7AL

United Kingdom 